\title{
A two-dimensional model for extensional motion of a pre-stressed incompressible elastic layer near cut-off frequencies
}

\author{
Aleksey V. Pichugin and Graham A. Rogerson * \\ Department of Computer and Mathematical Sciences, \\ University of Salford, Salford. M5 4WT. UK.
}

\begin{abstract}
A two-dimensional model for extensional motion of a pre-stressed incompressible elastic layer near its cut-off frequencies is derived. Leading order solutions for displacement and pressure are obtained in terms of the long wave amplitude by direct asymptotic integration. A governing equation, together with corrections for displacement and pressure, is derived from the second order problem. A novel feature of this (two-dimensional) hyperbolic governing equation is that, for certain pre-stressed states, time and one of the two (in-plane) spatial variables can change roles. Although whenever this phenomenon occurs the equation still remains hyperbolic, it is clearly not wave-like. The second order solution is completed by deriving a refined governing equation from the third order problem. Asymptotic consistency, in the sense that the dispersion relation associated with the two-dimensional model concurs with the appropriate order expansion of the three-dimensional relation at each order, is verified. The model has particular application to stationary thickness vibration of, or transient response to high frequency shock loading in, thin walled bodies.
\end{abstract}

\section{Introduction}

In this paper we seek to derive a lower dimensional model to describe long wave high frequency motion in a pre-stressed incompressible elastic layer. Specifically, a two-dimensional

*Author for correspondence 
model is derived through asymptotic integration of the three-dimensional equations for motion in the vicinity of the cut-off frequencies. The term high frequency is used to distinguish between long wave motion associated with the fundamental mode, this usually being termed long wave low frequency. Understanding of long wave high frequency motion is important for elucidation of the dynamic response of thin walled structures, especially the response to stationary thickness vibration or the transient response to high frequency shock loading. Additionally, this type of motion provides some contribution to the transient response to any kind of dynamic load. Amongst other noteworthy applications is fluid-structure interaction, particularly jumps in radiation power and first order resonances of high frequency Lamb waves in scattering, phenomena known to occur in the long wave high frequency region, see for example Kaplunov \& Markushevich (1993). We also note the possible dominance of motions of this type in problems involving fixed faces, such problems being characterised by the absence of fundamental modes, see for example Kaplunov (1995).

The specific constitutive model employed, namely an incompressible elastic solid, is motivated through the increasing industrial usage of rubber-like material. Such material, with its ability to withstand large deformation and support high external loads prior to failure, has proved highly beneficial for vibration control devices, see Sheridan et al. (1992). It is anticipated that the model presented will help elucidate the effects of pre-stress on the dynamic material characteristics of incompressible elastic solids. Although pre-stress may well be introduced during the manufacturing process, it is the aforementioned load supporting scenario that mainly motivates this work. In view of the important applications of this particular material model within such a context, a number a recent studies have focused on various aspects of wave propagation and vibration in plates, half-spaces and layered structures composed of such material, see for example Dowaikh \& Ogden (1991); Ogden \& Roxburgh (1993); Ogden \& Sotiropoulos (1995); Rogerson \& Fu (1995); Rogerson (1997); Rogerson \& Sandiford (1997).

Direct asymptotic integration, in the vicinity of cut-off frequencies, was seemingly first carried out within the framework of isotropic linear elasticity for stationary vibrations, see Achenbach (1969). Later, the connection between the direct asymptotic integration of static problems, see for example Goldenveiser (1962) and Goldenveiser (1966), and their dynamic counterparts was established, see Kaplunov et al. (1998), Kaplunov (1989) and Kaplunov (1990). All of the previously mentioned investigations are concerned with the application of direct asymptotic integration to isotropic elastic bodies. In the case of pre-stressed elastic bodies, relatively little work has been carried out in this area. Moreover, the work that has thus far been carried out has been restricted to the simplified plane strain case, see Kaplunov et al. (2000c) and Kaplunov et al. (2000b). The first of these concerns a long 
wave low frequency model, specifically associated with the fundamental mode of a plate at low wave number, whilst the second relates to the simplified plane strain analogous case to the present study.

This paper is organised as follows. In section 2 the three-dimensional equations for small amplitude time-dependent motion super-imposed upon a homogeneous static deformation in an incompressible elastic solids are reviewed. In order to simplify the analysis, attention is restricted to extensional motion and the appropriate dispersion relation and eigenfunctions, for the infinitesimal displacement components and pressure increment, are derived. In section three an asymptotic analysis of the dispersion relation in the vicinity of the non-zero cut-off frequencies is carried out. It is shown that for a layer composed of pre-stressed incompressible elastic material, two distinct families of cut-off frequencies exist. Expansions, giving frequency as a function of wave number, are established in each case. These asymptotic expansions are also used to establish the relative orders of infinitesimal displacements and pressure increment for motion in the vicinity of each family of cut-off frequencies. It is shown that in both cases one of the in-plane displacement components is asymptotically leading. The implication is that both families are associated with so-called thickness shear resonance, the constraint of incompressibility precluding thickness stretch resonance.

In section 4 appropriate scales for space and time are introduced and asymptotically approximate equations established. A perturbation scheme is set up and systems of equations, and corresponding boundary conditions, are obtained at each order. For both families of cut-off frequencies, the leading order displacement components and pressure increment are completely determined in terms of a single function. This function, termed the long wave amplitude, is a function of the two in-plane spatial variables and time. In order to derive a governing equation for the long wave amplitude the second order problem must be considered. This problem yields both the appropriate governing equation and a corrected solution for the displacement components and pressure increment in terms of a corrected long wave amplitude. A governing equation for the corrected long wave amplitude is derived from the third order problem.

An interesting feature of the governing equation for the leading order long wave amplitude is a possible switching of the roles between time and one of the (in-plane) spatial variables. Such a phenomenon will occur when one of the coefficients of the governing equation for the long wave amplitude changes sign. Moreover, this will be associated with certain pre-stressed states and may have an associated region of negative group velocity in the vicinity for the cut-off frequency. However, the governing equation will always remain hyperbolic. This contrasts with the plane strain case for which a similar switching of role necessarily changes the character of the governing equation from hyperbolic to elliptic and 
an associated region of negative group velocity always exists, see Kaplunov et al. (2000b). The paper is concluded in section 5 with illustrative numerical results for a Varga material.

\section{Mathematical preliminaries}

\subsection{Basic equations and notation}

We consider a body $\mathcal{B}$ composed of incompressible elastic material, possessing a natural unstressed configuration $\mathcal{B}_{0}$. A purely homogeneous static deformation is imposed on $\mathcal{B}_{0}$, resulting in a finitely deformed equilibrium state $\mathcal{B}_{e}$. Finally, a small amplitude timedependent motion is super-imposed on $\mathcal{B}_{e}$, with the final material configuration termed the current and denoted by $\mathcal{B}_{t}$. The linearised form of the equations of motion for a timedependent incremental motion super-imposed on a pre-stressed incompressible elastic material have previously been derived, see e.g. Dowaikh \& Ogden (1990), and take the form

$$
B_{\text {milk }} u_{k, l m}-p_{t, i}=\rho \ddot{u}_{i},
$$

in which $B_{\text {milk }}$ are components of the elasticity tensor, $\boldsymbol{u}$ is the infinitesimal displacement, $\rho$ the material density, $p_{t}=p_{t}(\mathbf{x}, t)$ a time dependent pressure increment, a comma and dot indicate differentiation with respect to a component of $\mathbf{x}$, the spatial variable in $\mathcal{B}_{e}$, and time $t$, respectively. We remark that in (2.1) use has been made of the summation convention. Throughout this paper we will always explicitly state occasions where use of this convention has been applied. A linearised measure of traction increment has also been previously obtained in the component form

$$
\tau_{i}=B_{m i l k} u_{k, l} n_{m}+\bar{p} u_{m, i} n_{m}-p_{t} n_{i},
$$

where $\bar{p}$ is a static pressure in $\mathcal{B}_{e}, \mathbf{n}$ the unit outward normal to a material surface in $\mathcal{B}_{e}$ and the summation convention is utilised, see for example Dowaikh \& Ogden (1990).

We now wish to investigate the case in which $\mathcal{B}$ forms a layer of finite thickness $2 h$ and infinite lateral extent in $\mathcal{B}_{e}$. An appropriate Cartesian coordinate system is chosen coincident with the principal axes of the primary deformation and such that the origin $O$ lies in the mid-plane, with $O x_{2}$ normal to the plane of the layer. For an incompressible (initially isotropic) elastic material all non-zero components of the elasticity tensor $\boldsymbol{B}$, referred to the above coordinate system, have one of forms $B_{i i j j}, B_{i j i j}$ and $B_{i j j i}$, where $B_{i i j j}=B_{j j i i}$ and $B_{i j j i}=B_{j i i j},(i, j \in\{1,2,3\})$, see Ogden (1984). Accordingly, the three linearised components of the equations of motion (2.1) may be written explicitly as

$$
\begin{aligned}
B_{1111} u_{1,11}+\left(B_{1122}+B_{2112}\right) u_{2,12}+ & \left(B_{1133}+B_{3113}\right) u_{3,13} \\
& +B_{2121} u_{1,22}+B_{3131} u_{1,33}-p_{t, 1}=\rho \ddot{u}_{1},
\end{aligned}
$$




$$
\begin{aligned}
\left(B_{2211}+B_{1221}\right) u_{1,12}+B_{2222} u_{2,22}+ & \left(B_{2233}+B_{3223}\right) u_{3,23} \\
& +B_{1212} u_{2,11}+B_{3232} u_{2,33}-p_{t, 2}=\rho \ddot{u}_{2}, \\
\left(B_{3311}+B_{1331}\right) u_{1,13}+\left(B_{3322}+B_{2332}\right) & u_{2,23}+B_{3333} u_{3,33} \\
& +B_{1313} u_{3,11}+B_{2323} u_{3,22}-p_{t, 3}=\rho \ddot{u}_{3} .
\end{aligned}
$$

For a layer with outward unit normal to the upper surface in $\mathcal{B}_{e}$ given by $\boldsymbol{n}=\delta_{i 2}$, the components of incremental surface traction (2.2) take the appropriate forms

$$
\begin{aligned}
\tau_{1} & =B_{2121} u_{1,2}+\left(B_{2112}+\bar{p}\right) u_{2,1}, \\
\tau_{2} & =B_{2211} u_{1,1}+\left(B_{2222}+\bar{p}\right) u_{2,2}+B_{2233} u_{3,3}-p_{t}, \\
\tau_{3} & =\left(B_{2332}+\bar{p}\right) u_{2,3}+B_{2323} u_{3,2} .
\end{aligned}
$$

The equations of motion (2.3)-(2.5) must be solved in conjunction with the linearised incompressibility constraint $u_{1,1}+u_{2,2}+u_{3,3}=0$. Solutions of these equations are now sought in the form of the traveling waves

$$
\left(u_{1}, u_{2}, u_{3}, p_{t}\right)=\left(U_{1}, U_{2}, U_{3}, k P\right) e^{k q x_{2}} e^{i k\left(x_{1} c_{\theta}+x_{3} s_{\theta}-v t\right)},
$$

within which $k$ is the wave number, $v$ is the phase speed, $q$ is to be determined and $s_{\theta}=\sin \theta$, $c_{\theta}=\cos \theta$, where $\theta$ is the angle of wave propagation relative to the $O x_{1}$ axis. Kaplunov et al. (2000b) have previously analysed the corresponding plane-strain problem, we therefore seek to generalise the problem to the three-dimensional case and tacitly assume that $s_{\theta} \neq 0$ and $c_{\theta} \neq 0$. If solutions of the form (2.9) are inserted into equations (2.3)-(2.5), and the linearised incompressibility condition, a system of four homogeneous equations is obtained. This system will have non-trivial solutions provided

$$
\gamma_{21} \gamma_{23} q^{6}+\left(\left(\gamma_{21}+\gamma_{23}\right) \bar{v}^{2}-c_{1}\right) q^{4}+\left(\bar{v}^{4}-c_{2} \bar{v}^{2}+c_{3}\right) q^{2}-\left(\bar{v}^{2}-c_{4}\right)\left(\bar{v}^{2}-c_{5}\right)=0,
$$

in which the scaled wave speed $\bar{v}^{2} \equiv \rho v^{2}$,

$$
\begin{aligned}
c_{1}= & \left(2 \beta_{23} \gamma_{21}+\gamma_{23} \gamma_{31}\right) s_{\theta}^{2}+\left(2 \beta_{12} \gamma_{23}+\gamma_{21} \gamma_{13}\right) c_{\theta}^{2}, \\
c_{2}= & \left(2 \beta_{23}+\gamma_{21}+\gamma_{31}\right) s_{\theta}^{2}+\left(2 \beta_{12}+\gamma_{23}+\gamma_{13}\right) c_{\theta}^{2}, \\
c_{3}= & \left(4 \beta_{12} \beta_{23}+\gamma_{21} \gamma_{12}+\gamma_{23} \gamma_{32}+\gamma_{13} \gamma_{31}-\mu_{13}^{2}\right) s_{\theta}^{2} c_{\theta}^{2} \\
& \quad+\left(2 \beta_{23} \gamma_{31}+\gamma_{21} \gamma_{32}\right) s_{\theta}^{4}+\left(2 \beta_{12} \gamma_{13}+\gamma_{23} \gamma_{12}\right) c_{\theta}^{4}, \\
c_{4}= & \gamma_{32} s_{\theta}^{2}+\gamma_{12} c_{\theta}^{2}, \quad c_{5}=\gamma_{31} s_{\theta}^{4}+2 \beta_{13} s_{\theta}^{2} c_{\theta}^{2}+\gamma_{13} c_{\theta}^{4},
\end{aligned}
$$

and

$$
\begin{gathered}
\gamma_{i j}=B_{i j i j}, \quad i \neq j, \quad 2 \beta_{i j}=2 \beta_{j i}=B_{i i i i}+B_{j j j j}-2 B_{i i j j}-2 B_{i j j i}, \quad i<j, \\
\mu_{i j}=\beta_{i j}-\beta_{i k}-\beta_{j k}, \quad i<j, \quad k \notin\{i, j\}, \quad i, j, k \in\{1,2,3\} .
\end{gathered}
$$


Apart from relating the parameter $q$ and scaled wave speed $\bar{v}$, the secular equation (2.10) may be used to establish some necessary conditions on possible values of material parameters. In an attempt to ensure physically reasonable response, we therefore tacitly assume that the speeds of all associated body waves are real. In the present context we only need to consider possible restrictions on $\gamma_{13}$ and $\gamma_{31}$. To do this we consider waves propagating in the $\left(x_{1}, x_{3}\right)$ plane. Such wave solutions may be obtained by setting $q=0$ in $(2.9)$ and equation (2.10) then reduces to

$$
\left(\bar{v}^{2}-c_{4}\right)\left(\bar{v}^{2}-c_{5}\right)=0 .
$$

The solutions of (2.11) yield the speeds of the two shear waves associated with propagation along $(\cos \theta, 0, \sin \theta)$. In order that these two wave speeds remain real for all $\theta$ we necessarily require that

$$
\gamma_{12}>0, \quad \gamma_{32}>0, \quad \gamma_{13}>0, \quad \gamma_{31}>0 .
$$

\subsection{Dispersion relation}

If the three (in general) distinct (non-zero) roots of the bi-cubic equation (2.10) are denoted by $q_{1}^{2}, q_{2}^{2}$ and $q_{3}^{2}$, the solutions of $u_{1}, u_{2}, u_{3}$ and $p_{t}$ are obtainable as linear combinations of the six linearly independent solutions. However, in this paper we restrict attention to the case for which $u_{2}$ vanishes at $x_{2}=0$, solutions for $u_{1}, u_{2}, u_{3}$ and $p_{t}$ then being obtainable as linear combinations of only three linearly independent solutions. This type of motion is usually referred to as extensional or symmetric motion and is equivalent to placing a layer of thickness $h$ on a rigid foundation. The equations of motion and incompressibility condition may be used to establish solutions in terms of only 3 disposable constants, thus

$$
\begin{array}{ll}
u_{1}=\sum_{m=1}^{3} \frac{i q_{m} \mathcal{U}_{1}\left(q_{m}, \bar{v}\right) c_{\theta}}{\mathcal{V}\left(q_{m}, \bar{v}\right)} C_{m}\left(x_{2}\right) U_{2}^{(m)}, & u_{2}=\sum_{m=1}^{3} S_{m}\left(x_{2}\right) U_{2}^{(m)}, \\
u_{3}=\sum_{m=1}^{3} \frac{i q_{m} \mathcal{U}_{3}\left(q_{m}, \bar{v}\right) s_{\theta}}{\mathcal{V}\left(q_{m}, \bar{v}\right)} C_{m}\left(x_{2}\right) U_{2}^{(m)}, & p_{t}=\sum_{m=1}^{3} \frac{q_{m} \mathcal{P}\left(q_{m}, \rho v^{2}\right)}{\mathcal{V}\left(q_{m}, \bar{v}\right)} C_{m}\left(x_{2}\right) U_{2}^{(m)},
\end{array}
$$

within which $S_{m}\left(x_{2}\right)=\sinh \left(k q_{m} x_{2}\right), C_{m}\left(x_{2}\right)=\cosh \left(k q_{m} x_{2}\right)$ and

$$
\begin{aligned}
\mathcal{U}_{1}\left(q_{m}, \bar{v}\right)= & \gamma_{23} q_{m}^{2}+\mu_{12} s_{\theta}^{2}-\gamma_{13} c_{\theta}^{2}+\bar{v}^{2}, \quad \mathcal{U}_{3}\left(q_{m}, \bar{v}\right)=\gamma_{21} q_{m}^{2}-\gamma_{31} s_{\theta}^{2}+\mu_{23} c_{\theta}^{2}+\bar{v}^{2} \\
\mathcal{P}\left(q_{m}, \bar{v}\right)= & \mathcal{U}_{1}\left(q_{m}, \bar{v}\right) \mathcal{U}_{3}\left(q_{m}, \bar{v}\right)+\left(B_{2233}+B_{2332}-B_{1133}-B_{1331}\right) \mathcal{U}_{1}\left(q_{m}, \bar{v}\right) c_{\theta}^{2} \\
& +\left(B_{1122}+B_{1221}-B_{1133}-B_{1331}\right) \mathcal{U}_{3}\left(q_{m}, \bar{v}\right) s_{\theta}^{2} \\
\mathcal{V}\left(q_{m}, \bar{v}\right)= & \left(\gamma_{21} s_{\theta}^{2}+\gamma_{23} c_{\theta}^{2}\right) q_{m}^{2}+\bar{v}^{2}-c_{5} .
\end{aligned}
$$

It is worth noting that in obtaining of the above representation of $\mathcal{P}\left(q_{m}, \bar{v}\right)$ use has been made of the fact that

$$
q_{m}^{2} \mathcal{U}_{1}\left(q_{m}, \bar{v}\right) \mathcal{U}_{3}\left(q_{m}, \bar{v}\right)=-\left(\mu_{13} q^{2}+\gamma_{32} s_{\theta}^{2}+\gamma_{12} c_{\theta}^{2}-\bar{v}^{2}\right) \mathcal{V}\left(q_{m}, \bar{v}\right),
$$


which may be established with use of (2.10). Corresponding representations of the traction increments may be found by inserting the form of displacement components given in (2.13) into equations (2.6)-(2.8), yielding

$$
\begin{aligned}
\frac{\tau_{1}}{i k} & =\sum_{m=1}^{3} \frac{\mathcal{T}_{1}\left(q_{m}, \bar{v}\right) c_{\theta}}{\mathcal{V}\left(q_{m}, \bar{v}\right)} S_{m}\left(x_{2}\right) \bar{U}_{2}^{(m)}, \\
\frac{\tau_{2}}{k} & =\sum_{m=1}^{3} \frac{q_{m} \mathcal{T}_{2}\left(q_{m}, \bar{v}\right)}{\mathcal{V}\left(q_{m}, \bar{v}\right)} C_{m}\left(x_{2}\right) \bar{U}_{2}^{(m)}, \\
\frac{\tau_{3}}{i k} & =\sum_{m=1}^{3} \frac{\mathcal{T}_{3}\left(q_{m}, \bar{v}\right) s_{\theta}}{\mathcal{V}\left(q_{m}, \bar{v}\right)} S_{m}\left(x_{2}\right) \bar{U}_{2}^{(m)},
\end{aligned}
$$

where $\bar{U}_{n}^{(m)}=U_{n}^{(m)} \exp \left(i k\left(x_{1} c_{\theta}+x_{3} s_{\theta}-v t\right)\right)$ and

$$
\begin{aligned}
\mathcal{T}_{1}\left(q_{m}, \bar{v}\right)= & \gamma_{21} \mathcal{U}_{1}\left(q_{m}, \bar{v}\right) q_{m}^{2}+\left(\gamma_{21}-\sigma_{2}\right) \mathcal{V}\left(q_{m}, \bar{v}\right) \\
\mathcal{T}_{2}\left(q_{m}, \bar{v}\right)= & \left(\gamma_{21}-\sigma_{2}-\mu_{13}\right) \mathcal{U}_{1}\left(q_{m}, \bar{v}\right) c_{\theta}^{2}-\mathcal{U}_{1}\left(q_{m}, \bar{v}\right) \mathcal{U}_{3}\left(q_{m}, \bar{v}\right) \\
& +\left(\gamma_{23}-\sigma_{2}-\mu_{13}\right) \mathcal{U}_{3}\left(q_{m}, \bar{v}\right) s_{\theta}^{2}, \\
\mathcal{T}_{3}\left(q_{m}, \bar{v}\right)= & \gamma_{23} \mathcal{U}_{3}\left(q_{m}, \bar{v}\right) q_{m}^{2}+\left(\gamma_{23}-\sigma_{2}\right) \mathcal{V}\left(q_{m}, \bar{v}\right) .
\end{aligned}
$$

A dispersion relation associated with infinitesimal harmonic wave propagation in the layer is now derived from equations (2.14) by applying traction free boundary conditions on each free surface to obtain $\tau_{1}=\tau_{2}=\tau_{3}=0$ at $x_{2}= \pm h$, thus

$$
\begin{gathered}
\sum_{m=1}^{3} \frac{\mathcal{T}_{1}\left(q_{m}, \bar{v}\right)}{\mathcal{V}\left(q_{m}, \bar{v}\right)} S_{m}(h) U_{2}^{(m)}=0, \\
\sum_{m=1}^{3} \frac{q_{m} \mathcal{T}_{2}\left(q_{m}, \bar{v}\right)}{\mathcal{V}\left(q_{m}, \bar{v}\right)} C_{m}(h) U_{2}^{(m)}=0, \\
\sum_{m=1}^{3} \frac{\mathcal{T}_{3}\left(q_{m}, \bar{v}\right)}{\mathcal{V}\left(q_{m}, \bar{v}\right)} S_{m}(h) U_{2}^{(m)}=0 .
\end{gathered}
$$

The condition that the system of equations (2.15) admit a non-trivial solution gives rise to the dispersion relation associated with extensional waves, taking the form

$$
\left|\begin{array}{ccc}
\mathcal{T}_{1}\left(q_{1}, \bar{v}\right) S_{1}(h) & \mathcal{T}_{1}\left(q_{2}, \bar{v}\right) S_{2}(h) & \mathcal{T}_{1}\left(q_{3}, \bar{v}\right) S_{3}(h) \\
q_{1} \mathcal{T}_{2}\left(q_{1}, \bar{v}\right) C_{1}(h) & q_{2} \mathcal{T}_{2}\left(q_{2}, \bar{v}\right) C_{2}(h) & q_{3} \mathcal{T}_{2}\left(q_{3}, \bar{v}\right) C_{3}(h) \\
\mathcal{T}_{3}\left(q_{1}, \bar{v}\right) S_{1}(h) & \mathcal{T}_{3}\left(q_{2}, \bar{v}\right) S_{2}(h) & \mathcal{T}_{3}\left(q_{3}, \bar{v}\right) S_{3}(h)
\end{array}\right|=0,
$$

in which common non-zero multipliers have been omitted. Evaluating the determinant in equation (2.16) and dividing by $C_{1}(h) C_{2}(h) C_{3}(h)$ leads to an explicit representation which, after a little algebraic manipulation, is given by

$$
\begin{array}{r}
\left(q_{2}^{2}-q_{3}^{2}\right) \mathcal{T}_{2}\left(q_{1}, \bar{v}\right) \mathcal{H}\left(q_{2}, q_{3}, \bar{v}\right) q_{1} T_{2}(h) T_{3}(h)-\left(q_{1}^{2}-q_{3}^{2}\right) \mathcal{T}_{2}\left(q_{2}, \bar{v}\right) \mathcal{H}\left(q_{1}, q_{3}, \bar{v}\right) q_{2} T_{1}(h) T_{3}(h) \\
+\left(q_{1}^{2}-q_{2}^{2}\right) \mathcal{T}_{2}\left(q_{3}, \bar{v}\right) \mathcal{H}\left(q_{1}, q_{2}, \bar{v}\right) q_{3} T_{1}(h) T_{2}(h)=0,
\end{array}
$$


in which $T_{m}(h)=\tanh \left(k q_{m} h\right), m \in\{1,2,3\}$,

$$
\begin{aligned}
\mathcal{H}\left(q_{i}, q_{j}, \bar{v}\right)= & \gamma_{21} \gamma_{23} \mathcal{H}_{1}(\bar{v}) q_{i}^{2} q_{j}^{2}+\left(\bar{v}^{2}-c_{5}\right)\left(\gamma_{21} \gamma_{23}\left(\gamma_{23}-\gamma_{21}\right)\left(q_{i}^{2}+q_{j}^{2}\right)-\mathcal{H}_{2}(\bar{v})\right), \\
\mathcal{H}_{1}(\bar{v})= & \left(\gamma_{23}-\gamma_{21}\right) \bar{v}^{2}-\left(\gamma_{21}\left(\mu_{12}-\gamma_{23}+\gamma_{21}\right)+\gamma_{23} \gamma_{31}\right) s_{\theta}^{2} \\
& \quad+\left(\gamma_{23}\left(\mu_{23}+\gamma_{23}-\gamma_{21}\right)+\gamma_{21} \gamma_{13}\right) c_{\theta}^{2}, \\
\mathcal{H}_{2}(\bar{v})= & \gamma_{23}\left(\gamma_{21}-\sigma_{2}\right)\left(\mu_{23} c_{\theta}^{2}-\gamma_{31} s_{\theta}^{2}+\bar{v}^{2}\right)-\gamma_{21}\left(\gamma_{23}-\sigma_{2}\right)\left(\mu_{12} s_{\theta}^{2}-\gamma_{13} c_{\theta}^{2}+\bar{v}^{2}\right),
\end{aligned}
$$

with $\mathcal{T}_{2}\left(q_{m}, \bar{v}\right)$ defined immediately below equations (2.14). We note that the dispersion relation was seemingly first derived, in slightly different notation, by Rogerson \& Sandiford (1999).

\section{Analysis of the dispersion relation}

\subsection{Approximation near cut-off frequencies}

We now seek approximations for the frequencies, as functions of wave number, in the long wave high frequency motion regime, i.e. in the vicinity of the non-zero cut-off frequencies. It is known that for this type of motion $\bar{v} \rightarrow \infty$ as $k h \rightarrow 0$, see for example Rogerson (1997). Analysis of the relative orders of the coefficients of the secular equation (2.10) suggests that two roots are of order $O\left(\bar{v}^{2}\right)$, with the third root of order $O(1)$. More specifically, approximations for $q_{1}^{2}, q_{2}^{2}$ and $q_{3}^{2}$ are given by

$$
\begin{aligned}
& q_{1}^{2}=-\frac{\bar{v}^{2}}{\gamma_{21}}+\frac{\mathcal{Q}_{1 s}^{(0)} s_{\theta}^{2}+\mathcal{Q}_{1 c}^{(0)} c_{\theta}^{2}}{\gamma_{21}}-\left(\mathcal{Q}_{1 s}^{(-2)} s_{\theta}^{2}+\mathcal{Q}_{1 c}^{(-2)} c_{\theta}^{2}\right) \frac{c_{\theta}^{2}}{\bar{v}^{2}}+O\left(\bar{v}^{-4}\right), \\
& q_{2}^{2}=1+O\left(\bar{v}^{-2}\right), \\
& q_{3}^{2}=-\frac{\bar{v}^{2}}{\gamma_{23}}+\frac{\mathcal{Q}_{3 s}^{(0)} s_{\theta}^{2}+\mathcal{Q}_{3 c}^{(0)} c_{\theta}^{2}}{\gamma_{23}}-\left(\mathcal{Q}_{3 s}^{(-2)} s_{\theta}^{2}+\mathcal{Q}_{3 c}^{(-2)} c_{\theta}^{2}\right) \frac{s_{\theta}^{2}}{\bar{v}^{2}}+O\left(\bar{v}^{-4}\right),
\end{aligned}
$$

in which

$$
\begin{aligned}
\mathcal{Q}_{1 c}^{(0)} & =2 \beta_{12}-\gamma_{21}, \quad \mathcal{Q}_{1 s}^{(0)}=\gamma_{31}, & & \mathcal{Q}_{3 c}^{(0)}=\gamma_{13}, \quad \mathcal{Q}_{3 s}^{(0)}=2 \beta_{23}-\gamma_{23}, \\
\mathcal{Q}_{1 s}^{(-2)} & =\gamma_{31}-\gamma_{32}+\frac{\left(\mu_{13}+\gamma_{21}\right)^{2}}{\gamma_{23}-\gamma_{21}}, & & \mathcal{Q}_{3 c}^{(-2)}=\gamma_{13}-\gamma_{12}-\frac{\left(\mu_{13}+\gamma_{23}\right)^{2}}{\gamma_{23}-\gamma_{21}} \\
\mathcal{Q}_{1 c}^{(-2)} & =2 \beta_{12}-\gamma_{21}-\gamma_{12}, & & \mathcal{Q}_{3 s}^{(-2)}=2 \beta_{23}-\gamma_{23}-\gamma_{32} .
\end{aligned}
$$

Further investigation will also require expansions for $q_{1}, q_{2}$ and $q_{3}$, these can be derived from (3.1), yielding

$$
\begin{aligned}
& q_{1}=\frac{i \bar{v}}{\sqrt{\gamma_{21}}}-\frac{i\left(\mathcal{Q}_{1 s}^{(0)} s_{\theta}^{2}+\mathcal{Q}_{1 c}^{(0)} c_{\theta}^{2}\right)}{2 \sqrt{\gamma_{21}} \bar{v}}+O\left(\bar{v}^{-3}\right), \\
& q_{2}=1+O\left(\bar{v}^{-2}\right) \\
& q_{3}=\frac{i \bar{v}}{\sqrt{\gamma_{23}}}-\frac{i\left(\mathcal{Q}_{3 s}^{(0)} s_{\theta}^{2}+\mathcal{Q}_{3 c}^{(0)} c_{\theta}^{2}\right)}{2 \sqrt{\gamma_{23}} \bar{v}}+O\left(\bar{v}^{-3}\right) .
\end{aligned}
$$


The secular equation (2.10) may also be used to obtain expansions for the scaled speed $\bar{v}$ as function of $q$. In view of the fact that the $\bar{v} \rightarrow \infty$ as $k h \rightarrow 0$, it can be deduced that the only speeds of interest are those associated with the $q_{1}$ and $q_{3}$, see expansions (3.1), and these speeds are given by the following relations

$$
\begin{aligned}
& \bar{v}_{1}^{2}=-\gamma_{21} q_{1}^{2}+\mathcal{Q}_{1 s}^{(0)} s_{\theta}^{2}+\mathcal{Q}_{1 c}^{(0)} c_{\theta}^{2}+\left(\mathcal{Q}_{1 s}^{(-2)} s_{\theta}^{2}+\mathcal{Q}_{1 c}^{(-2)} c_{\theta}^{2}\right) \frac{c_{\theta}^{2}}{q_{1}^{2}}+O\left(q_{1}^{-4}\right), \\
& \bar{v}_{3}^{2}=-\gamma_{23} q_{3}^{2}+\mathcal{Q}_{3 s}^{(0)} s_{\theta}^{2}+\mathcal{Q}_{3 c}^{(0)} c_{\theta}^{2}+\left(\mathcal{Q}_{3 s}^{(-2)} s_{\theta}^{2}+\mathcal{Q}_{3 c}^{(-2)} c_{\theta}^{2}\right) \frac{s_{\theta}^{2}}{q_{3}^{2}}+O\left(q_{3}^{-4}\right),
\end{aligned}
$$

the speed associated with $q_{2}$ being of $O(1)$. The existence of one value of $q^{2}$ that is $O(1)$ is a direct consequence of imposing of the incompressibility constraint, which disables thickness stretch resonance. For an example of a similar leading order asymptotic analysis for an unconstrained linear transversely isotropic material, for which thickness shear resonance exists, the reader is referred to Kaplunov et al. (2000a).

At this stage it is convenient to introduce a small non-dimensional parameter $\eta$, defined as the ratio of a layer half-thickness $h$ to a typical wavelength $l$, so $\eta=h / l=k h$. Now by inserting approximations (3.1) into the dispersion relation (2.17) we obtain the leading order approximation of the dispersion relation, namely

$$
\begin{gathered}
i A_{1} T_{2}(h) T_{3}(h)-\bar{v}^{3} T_{1}(h) T_{3}(h)+i A_{3} T_{1}(h) T_{2}(h) \sim 0, \\
A_{1}=\frac{\left(2 \gamma_{21}-\sigma_{2}\right)^{2} c_{\theta}^{2}}{\sqrt{\gamma_{21}}}, \quad A_{3}=\frac{\left(2 \gamma_{23}-\sigma_{2}\right)^{2} s_{\theta}^{2}}{\sqrt{\gamma_{23}}} .
\end{gathered}
$$

Hereafter, it is assumed that the functions of material parameters $A_{1}, A_{3}$ are generally $O(1)$. Since $q_{2}=1+O\left(\bar{v}^{-2}\right)$, see $(3.2)$, it is clear that $T_{2}(h)=O\left(\bar{v}^{-1}\right)$. This observation suggests that terms on the left-hand side of the relation (3.5) can be asymptotically balanced only if $T_{1}(h)=O\left(\bar{v}^{-4}\right)$ or $T_{3}(h)=O\left(\bar{v}^{-4}\right)$. In fact, each of these asymptotic relations corresponds to the harmonics associated with one of the two possible families of cut-off frequencies.

We shall consider first the case in which $T_{1}(h)=O\left(\bar{v}^{-4}\right)$, the asymptotic order of $T_{1}(h)$ then implying the following expansions

$$
k q_{1} h=i\left(\pi n+\phi_{1}^{e(4)} \eta^{4}+O\left(\eta^{6}\right)\right), \quad T_{1}(h)=i \phi_{1}^{e(4)} \eta^{4}+O\left(\eta^{6}\right),
$$

in which the function of material parameters $\phi_{1}^{e(4)}$ is to be determined. In this case an approximation for the wave speed is given by (3.3). We may invoke relations (3.2), and the definition of $T_{m}(h)$ given immediately after equation (2.17), to show that

$$
\begin{aligned}
q_{1} & =\frac{i \Lambda_{1}^{e}}{\sqrt{\gamma_{21}} \eta}+O\left(\eta^{3}\right), & q_{2}=1+O\left(\eta^{2}\right), & q_{3}=\frac{i \Lambda_{1}^{e}}{\sqrt{\gamma_{23}} \eta}+O(\eta), \\
\bar{v} & =\frac{\Lambda_{1}^{e}}{\eta}+O(\eta), & T_{2}(h)=\eta+O\left(\eta^{3}\right), & T_{3}(h)=O(1),
\end{aligned}
$$


within which $\Lambda_{1}^{e}=\sqrt{\gamma_{21}} \pi n, n=1,2,3 \ldots$ are the associated cut-off frequencies. It is now possible to determine an explicit expression for the function $\phi_{1}^{e(4)}$ by utilising expansions (3.6) and (3.7), together with the leading order approximation of dispersion relation (3.5), to obtain

$$
\phi_{1}^{e(4)}=\frac{A_{1}}{\left(\Lambda_{1}^{e}\right)^{3}} .
$$

The desired approximation of scaled frequency $\bar{\omega}=\bar{v} \eta$ of long wave high frequency motion is finally derived from the wave speed approximation (3.3) and the expansion for $q_{1}$ given in (3.6), yielding

$$
\begin{aligned}
& \bar{\omega}^{2}=\left(\Lambda_{1}^{e}\right)^{2}+\left(\mathcal{E}_{1 s}^{(2)} s_{\theta}^{2}+\mathcal{E}_{1 c}^{(2)} c_{\theta}^{2}\right) \eta^{2}+\left(\mathcal{E}_{1 s}^{(4)} s_{\theta}^{2}+\mathcal{E}_{1 c}^{(4)} c_{\theta}^{2}\right) c_{\theta}^{2} \eta^{4}+O\left(\eta^{6}\right), \\
& \mathcal{E}_{1 s}^{(2)}=\mathcal{Q}_{1 s}^{(0)}, \quad \mathcal{E}_{1 c}^{(2)}=\mathcal{Q}_{1 c}^{(0)}, \\
& \mathcal{E}_{1 s}^{(4)}=\frac{2\left(2 \gamma_{21}-\sigma_{2}\right)^{2}-\gamma_{21} \mathcal{Q}_{1 s}^{(-2)}}{\left(\Lambda_{1}^{e}\right)^{2}}, \quad \mathcal{E}_{1 c}^{(4)}=\frac{2\left(2 \gamma_{21}-\sigma_{2}\right)^{2}-\gamma_{21} \mathcal{Q}_{1 c}^{(-2)}}{\left(\Lambda_{1}^{e}\right)^{2}} .
\end{aligned}
$$

We shall now consider the second case $T_{3}(h)=O\left(\bar{v}^{-4}\right)$. Following the same procedure we expand $q_{3}$ to obtain

$$
k q_{3} h=i\left(\pi n+\phi_{3}^{e(4)} \eta^{4}+O\left(\eta^{6}\right)\right), \quad T_{3}(h)=i \phi_{3}^{e(4)} \eta^{4}+O\left(\eta^{6}\right),
$$

with the wave speed given by expansion (3.4). Introducing the second family of cut-off frequencies $\Lambda_{3}^{e}=\sqrt{\gamma_{23}} \pi n, n=1,2,3 \ldots$, and utilising relations (3.2), the following approximations can be derived

$$
\begin{array}{rlrl}
q_{3} & =\frac{i \Lambda_{3}^{e}}{\sqrt{\gamma_{23}} \eta}+O\left(\eta^{3}\right), & q_{2}=1+O\left(\eta^{2}\right), & q_{1}=\frac{i \Lambda_{3}^{e}}{\sqrt{\gamma_{21}} \eta}+O(\eta), \\
\bar{v} & =\frac{\Lambda_{3}^{e}}{\eta}+O(\eta), \quad T_{2}(h)=\eta+O\left(\eta^{3}\right), \quad T_{1}(h)=O(1) .
\end{array}
$$

The function $\phi_{3}^{e(4)}$ is now determined from (3.5), (3.10) and (3.11), thus

$$
\phi_{3}^{e(4)}=\frac{A_{3}}{\left(\Lambda_{3}^{e}\right)^{3}} .
$$

Finally, through use of (3.4) and (3.10), the approximation of the scaled frequency associated with the second family of cut-off frequencies is given by

$$
\begin{aligned}
& \bar{\omega}^{2}=\left(\Lambda_{3}^{e}\right)^{2}+\left(\mathcal{E}_{3 s}^{(2)} s_{\theta}^{2}+\mathcal{E}_{3 c}^{(2)} c_{\theta}^{2}\right) \eta^{2}+\left(\mathcal{E}_{3 s}^{(4)} s_{\theta}^{2}+\mathcal{E}_{3 c}^{(4)} c_{\theta}^{2}\right) s_{\theta}^{2} \eta^{4}+O\left(\eta^{6}\right), \\
& \mathcal{E}_{3 s}^{(2)}=\mathcal{Q}_{3 s}^{(0)}, \quad \mathcal{E}_{3 c}^{(2)}=\mathcal{Q}_{3 c}^{(0)}, \\
& \mathcal{E}_{3 s}^{(4)}=\frac{2\left(2 \gamma_{23}-\sigma_{2}\right)^{2}-\gamma_{23} \mathcal{Q}_{3 s}^{(-2)}}{\left(\Lambda_{3}^{e}\right)^{2}}, \quad \mathcal{E}_{3 c}^{(4)}=\frac{2\left(2 \gamma_{23}-\sigma_{2}\right)^{2}-\gamma_{23} \mathcal{Q}_{3 c}^{(-2)}}{\left(\Lambda_{3}^{e}\right)^{2}} .
\end{aligned}
$$

\subsection{Relative orders of displacements}

Construction of the lower dimensional model requires knowledge of the relative orders of particle displacements for long wave high frequency motion. The boundary conditions $(2.15)_{1,3}$ 
may be used to represent $U_{2}^{(k)}, k \in\{1,2,3\}$, in terms of single constant $U_{2}^{(0)}$, yielding

$$
U_{2}^{(k)}=\frac{\left(q_{i}^{2}-q_{j}^{2}\right) \mathcal{H}\left(q_{i}, q_{j}, \bar{v}\right) \mathcal{V}\left(q_{k}, \bar{v}\right)}{S_{k}(h)} U_{2}^{(0)}, \quad i<j, \quad k \notin\{i, j\}, \quad i, j, k \in\{1,2,3\} .
$$

Substituting (3.14) into the displacement relations (2.13) we may compare the relative order of displacement components. To do this we will have to estimate the orders of all functions occurring in (2.13) and (3.14), and we begin with

$$
S_{2}\left(x_{2}\right)=\eta \frac{x_{2}}{h}+O\left(\eta^{3}\right), \quad C_{2}\left(x_{2}\right)=1+O\left(\eta^{2}\right) .
$$

The remaining functions must be analysed separately for the motions in the vicinity of each set of cut-off frequencies. It is also worth noting that subsequently we assume the value $x_{2} / h$ to be $O(1)$. In the first case $\left(\omega=\Lambda_{1}^{e}\right)$ the expansions (3.7) and (3.15), supplemented by

$$
\begin{gathered}
S_{1}\left(x_{2}\right)=i \sin \left(\frac{\Lambda_{1}^{e} x_{2}}{\sqrt{\gamma_{21}} h}\right)+O\left(\eta^{2}\right), \quad C_{1}\left(x_{2}\right)=i \cos \left(\frac{\Lambda_{1}^{e} x_{2}}{\sqrt{\gamma_{21}} h}\right)+O\left(\eta^{2}\right), \\
S_{3}\left(x_{2}\right)=i \sin \left(\frac{\Lambda_{1}^{e} x_{2}}{\sqrt{\gamma_{23}} h}\right)+O\left(\eta^{2}\right), \quad C_{3}\left(x_{2}\right)=i \cos \left(\frac{\Lambda_{1}^{e} x_{2}}{\sqrt{\gamma_{23}} h}\right)+O\left(\eta^{2}\right), \\
S_{1}(h)=i(-1)^{n} \phi_{1}^{e(4)} \eta^{4}+O\left(\eta^{6}\right),
\end{gathered}
$$

reveal that the orders of displacements are distributed as follows

$$
U_{1} \sim O(P), \quad U_{2} \sim \eta O(P), \quad U_{3} \sim \eta^{2} O(P) .
$$

We repeat this routine for the second case $\left(\omega=\Lambda_{3}^{e}\right)$, taking together

$$
\begin{gathered}
S_{1}\left(x_{2}\right)=i \sin \left(\frac{\Lambda_{3}^{e} x_{2}}{\sqrt{\gamma_{21}} h}\right)+O\left(\eta^{2}\right), \quad C_{1}\left(x_{2}\right)=i \cos \left(\frac{\Lambda_{3}^{e} x_{2}}{\sqrt{\gamma_{21}} h}\right)+O\left(\eta^{2}\right), \\
S_{3}\left(x_{2}\right)=i \sin \left(\frac{\Lambda_{3}^{e} x_{2}}{\sqrt{\gamma_{23}} h}\right)+O\left(\eta^{2}\right), \quad C_{3}\left(x_{2}\right)=i \cos \left(\frac{\Lambda_{3}^{e} x_{2}}{\sqrt{\gamma_{23}} h}\right)+O\left(\eta^{2}\right), \\
S_{3}(h)=i(-1)^{n} \phi_{3}^{e(4)} \eta^{4}+O\left(\eta^{6}\right),
\end{gathered}
$$

and approximations (3.11) to indicate that for the second family of cut-off frequencies

$$
U_{1} \sim \eta^{2} O(P), \quad U_{2} \sim \eta O(P), \quad U_{3} \sim O(P) .
$$

The results (3.16) and (3.17) clearly indicate that in each case the leading order displacement is one of the in-plane displacements. It is then inferred that there exist only so-called thickness shear resonance, the existence of thickness stretch resonance being precluded by the incompressibility constraint.

\section{Asymptotically approximate equations}

The deriving of the asymptotically approximate equations requires appropriate rescaling of the spatial and time variables as well as particle displacements and pressure increment. We 
choose scalings of the spatial coordinates so as to balance $x_{2}$ with half-thickness $h$ and $x_{1}, x_{3}$ with with a typical wave length $l$

$$
x_{1}=l \xi_{1}, \quad x_{2}=h \zeta=l \eta \zeta, \quad x_{3}=l \xi_{3} .
$$

Corresponding rescaling of time, displacements and pressure is best done separately for each family of cut-off frequencies, these then being examined each in turn.

\subsection{First family of the cut-off frequencies}

For the motions in the vicinity of the first family of cut-off frequencies a typical wave travels with the speed $v \sim \frac{\Lambda_{1}^{e}}{\eta \sqrt{\rho}}$, see scaled speed $\bar{v}$ approximation given in (3.7), covering the distance of a typical wave length $l$ in a time $t \sim l \frac{\eta \sqrt{\rho}}{\Lambda_{1}^{e}}$. Upon invoking the definition of the $\Lambda_{1}^{e}$, given immediately after (3.7), we therefore introduce the following scaling of time

$$
t=l \eta \sqrt{\frac{\rho}{\gamma_{21}}} \tau .
$$

The displacement components and incremental pressure are scaled according to their relative orders, indicated in (3.16), yielding

$$
\begin{gathered}
u_{m}\left(x_{1}, x_{2}, x_{3}, t\right)=l \eta^{m-1} u_{m}^{*}\left(\xi_{1}, \zeta, \xi_{3}, \tau\right), \quad m \in\{1,2,3\}, \\
p_{t}\left(x_{1}, x_{2}, x_{3}, t\right)=\gamma_{21} p_{t}^{*}\left(\xi_{1}, \zeta, \xi_{3}, \tau\right),
\end{gathered}
$$

in which superscript ${ }^{*}$ denotes non-dimensional quantities of the same asymptotic order.

The equations of motion (2.3)-(2.5) can now be formulated in terms of the rescaled non-dimensional variables, so that

$$
\begin{aligned}
\gamma_{21} u_{1, \zeta \zeta}^{*}+ & \left(\Lambda_{1}^{e}\right)^{2} u_{1}^{*}-\left\{\left(\Lambda_{1}^{e}\right)^{2} u_{1}^{*}+\gamma_{21} u_{1, \tau \tau}^{*}\right\}+\eta^{2}\left(B_{1111} u_{1, \xi_{1} \xi_{1}}^{*}+\gamma_{31} u_{1, \xi_{3} \xi_{3}}^{*}\right. \\
& \left.+\left(B_{1122}+B_{1221}\right) u_{2, \xi_{1} \zeta}^{*}-\gamma_{21} p_{t, \xi_{1}}^{*}\right)+\eta^{4}\left(B_{1133}+B_{1331}\right) u_{3, \xi_{1} \xi_{3}}^{*}=0 \\
B_{2222} u_{2, \zeta \zeta}^{*}+ & \left(\Lambda_{1}^{e}\right)^{2} u_{2}^{*}-\left\{\left(\Lambda_{1}^{e}\right)^{2} u_{2}^{*}+\gamma_{21} u_{2, \tau \tau}^{*}\right\}+\left(B_{1122}+B_{1221}\right) u_{1, \xi_{1} \zeta}^{*} \\
& -\gamma_{21} p_{t, \zeta}^{*}+\eta^{2}\left(\gamma_{12} u_{2, \xi_{1} \xi_{1}}^{*}+\gamma_{32} u_{2, \xi_{3} \xi_{3}}^{*}+\left(B_{2233}+B_{2332}\right) u_{3, \xi_{3} \zeta}^{*}\right)=0 \\
\gamma_{23} u_{3, \zeta \zeta}^{*}+ & \left(\Lambda_{1}^{e}\right)^{2} u_{3}^{*}-\left\{\left(\Lambda_{1}^{e}\right)^{2} u_{3}^{*}+\gamma_{21} u_{3, \tau \tau}^{*}\right\}+\left(B_{1133}+B_{1331}\right) u_{1, \xi_{1} \xi_{3}}^{*} \\
& +\left(B_{2233}+B_{2332}\right) u_{2, \xi_{3} \zeta}^{*}-\gamma_{21} p_{t, \xi_{3}}^{*}+\eta^{2}\left(\gamma_{13} u_{3, \xi_{1} \xi_{1}}^{*}+B_{3333} u_{3, \xi_{3} \xi_{3}}^{*}\right)=0
\end{aligned}
$$

in which we use ${ }_{, \xi_{1}},{ }_{,},,_{\xi_{3}}$ and ${ }_{, \tau}$ to denote differentiation with respect to $\xi_{1}, \zeta, \xi_{3}$ and $\tau$, respectively. These equations must be solved in conjunction with the appropriate form of the incompressibility condition, namely

$$
u_{1, \xi_{1}}^{*}+u_{2, \zeta}^{*}+\eta^{2} u_{3, \xi_{3}}^{*}=0
$$


and the incremental surface traction boundary conditions

$$
\begin{gathered}
\gamma_{21} u_{1, \zeta}^{*}+\eta^{2}\left(B_{1221}+\bar{p}\right) u_{2, \xi_{1}}^{*}=0 \quad \text { at } \zeta= \pm 1, \\
B_{1122} u_{1, \xi_{1}}^{*}+\left(B_{2222}+\bar{p}\right) u_{2, \zeta}^{*}-\gamma_{21} p_{t}^{*}+\eta^{2} B_{2233} u_{3, \xi_{3}}^{*}=0 \quad \text { at } \zeta= \pm 1, \\
\gamma_{23} u_{3, \zeta}^{*}+\left(B_{2332}+\bar{p}\right) u_{2, \xi_{3}}^{*}=0 \quad \text { at } \zeta= \pm 1 .
\end{gathered}
$$

We will seek solutions of the boundary value problem given by equations (4.4)-(4.6) and (4.7), subject to (4.8)-(4.10), in a form of the power series

$$
\left(u_{1}^{*}, u_{2}^{*}, u_{3}^{*}, p_{t}^{*}\right)=\sum_{n=0}^{m} \eta^{2 n}\left(u_{1}^{*(2 n)}, u_{2}^{*(2 n)}, u_{3}^{*(2 n)}, p_{t}^{*(2 n)}\right)+O\left(\eta^{2 m+2}\right) .
$$

The result of substituting solutions (4.11) into the equations (4.4)-(4.10) is a hierarchical system of equations and boundary conditions at various orders. To solve this system, it is also necessary to utilise the fact that for the motion in the vicinity of the cut-off frequencies

$$
\gamma_{21} u_{m, \tau \tau}^{*}+\left(\Lambda_{1}^{e}\right)^{2} u_{m}^{*} \sim \eta^{2} u_{m}^{*}, \quad m \in\{1,2,3\},
$$

which can be verified with (3.9). This allows us to conclude that the quantities within braces in the equations of motion (4.4)-(4.6) are, in fact, of order $O\left(\eta^{2}\right)$. Consequently, the asymptotic solutions to the system (4.4)-(4.10) are obtainable by solving a sequence of systems of essentially ordinary differential equations.

\subsubsection{Leading order problem}

At leading order our problem is given by the equations of motion

$$
\begin{aligned}
\gamma_{21} u_{1, \zeta \zeta}^{*(0)}+\left(\Lambda_{1}^{e}\right)^{2} u_{1}^{*(0)} & =0, \\
B_{2222} u_{2, \zeta \zeta}^{*(0)}+\left(\Lambda_{1}^{e}\right)^{2} u_{2}^{*(0)} & +\left(B_{1122}+B_{1221}\right) u_{1, \xi_{1} \zeta}^{*(0)}-\gamma_{21} p_{t, \zeta}^{*(0)}=0, \\
\gamma_{23} u_{3, \zeta \zeta}^{*(0)}+\left(\Lambda_{1}^{e}\right)^{2} u_{3}^{*(0)} & +\left(B_{1133}+B_{1331}\right) u_{1, \xi_{1} \xi_{3}}^{*(0)} \\
& +\left(B_{2233}+B_{2332}\right) u_{2, \xi_{3} \zeta}^{*(0)}-\gamma_{21} p_{t, \xi_{3}}^{*(0)}=0,
\end{aligned}
$$

and incompressibility condition

$$
u_{1, \xi_{1}}^{*(0)}+u_{2, \zeta}^{*(0)}=0,
$$

which must be solved subject to leading order boundary conditions

$$
\begin{gathered}
\gamma_{21} u_{1, \zeta}^{*(0)}=0 \quad \text { at } \zeta= \pm 1, \\
\left(B_{2222}+\bar{p}\right) u_{2, \zeta}^{*(0)}+B_{1122} u_{1, \xi_{1}}^{*(0)}-\gamma_{21} p_{t}^{*(0)}=0 \quad \text { at } \zeta= \pm 1, \\
\gamma_{23} u_{3, \zeta}^{*(0)}+\left(B_{2332}+\bar{p}\right) u_{2, \xi_{3}}^{*(0)}=0 \quad \text { at } \zeta= \pm 1 .
\end{gathered}
$$

The solution of the boundary value problem (4.13), (4.17) may be represented in the form

$$
u_{1}^{*(0)}=u_{1}^{*(0,0)} \cos \left(\frac{\Lambda_{1}^{e} \zeta}{\sqrt{\gamma_{21}}}\right)
$$


where the function with double superscript is independent of $\zeta$. Hereafter, all functions with a double superscript will be assumed independent of $\zeta$ and therefore functions only of $\xi_{1}, \xi_{3}$ and $\tau$. Specifically, the first superscript refers to the order of the approximation, explanation of the second is deferred until later. Substituting the solution for $u_{1}^{*(0)}$ from (4.20) into the incompressibility condition (4.16) we obtain a differential equation for $u_{2}^{*(0)}$, indicating that

$$
u_{2}^{*(0)}=u_{2}^{*(0,0)} \sin \left(\frac{\Lambda_{1}^{e} \zeta}{\sqrt{\gamma_{21}}}\right)+U_{2}^{*(0,0)}, \quad u_{2}^{*(0,0)}=-\frac{\sqrt{\gamma_{21}}}{\Lambda_{1}^{e}} u_{1, \xi_{1}}^{*(0,0)} .
$$

We now substitute the previously determined functions $u_{1}^{*(0)}$ and $u_{2}^{*(0)}$, see (4.20) and (4.21), into equation (4.14), which when solved subject to (4.18) yields

$$
\gamma_{21} p_{t}^{*(0)}=p_{t}^{*(0,0)} \cos \left(\frac{\Lambda_{1}^{e} \zeta}{\sqrt{\gamma_{21}}}\right)+\zeta P_{t}^{*(0,1)}+P_{t}^{*(0,0)},
$$

within which

$$
p_{t}^{*(0,0)}=\left(\gamma_{21}-B_{2222}+B_{1122}+B_{1221}\right) u_{1, \xi_{1}}^{*(0,0)}, \quad P_{t}^{*(0,1)}=\left(\Lambda_{1}^{e}\right)^{2} U_{2}^{*(0,0)},
$$

with the boundary condition (4.18) immediately revealing that

$$
U_{2}^{*(0,0)}=0, \quad P_{t}^{*(0,1)}=0, \quad P_{t}^{*(0,0)}=-\left(2 \gamma_{21}-\sigma_{2}\right) u_{1, \xi_{1}}^{*(0,0)} \cos \left(\frac{\Lambda_{1}^{e}}{\sqrt{\gamma_{21}}}\right) .
$$

The leading order term of the third displacement component $u_{3}^{*(0)}$ is governed by equation (4.15), subject to the boundary condition (4.19). In view of results (4.20), (4.21) and (4.22), we seek the solution of (4.15) in the form

$$
u_{3}^{*(0)}=v_{3}^{*(0,0)} \cos \left(\frac{\Lambda_{1}^{e} \zeta}{\sqrt{\gamma_{23}}}\right)+v_{3}^{*(0,1)} \sin \left(\frac{\Lambda_{1}^{e} \zeta}{\sqrt{\gamma_{23}}}\right)+u_{3}^{*(0,0)} \cos \left(\frac{\Lambda_{1}^{e} \zeta}{\sqrt{\gamma_{21}}}\right)+U_{3}^{*(0,0)},
$$

in which

$$
u_{3}^{*(0,0)}=\frac{\gamma_{21}\left(\gamma_{21}+\mu_{13}\right)}{\left(\gamma_{21}-\gamma_{23}\right)\left(\Lambda_{1}^{e}\right)^{2}} u_{1, \xi_{1} \xi_{3}}^{*(0,0)}, \quad U_{3}^{*(0,0)}=-\frac{2 \gamma_{21}-\sigma_{2}}{\left(\Lambda_{1}^{e}\right)^{2}} \cos \left(\frac{\Lambda_{1}^{e}}{\sqrt{\gamma_{21}}}\right) u_{1, \xi_{1} \xi_{3}}^{*(0,0)},
$$

and a glance at the boundary condition (4.19) shows that it can only be satisfied when

$$
v_{3}^{*(0,0)}=0, \quad v_{3}^{*(0,1)}=0 .
$$

We note that for the extensional (symmetric) motion the in-plane displacements $u_{1}^{*}$, $u_{3}^{*}$ and pressure $p_{t}^{*}$ vary only as symmetric functions of the normal coordinate $\zeta$, whereas the normal displacement $u_{2}^{*}$ is an anti-symmetric function. Our leading order solutions (4.20)-(4.23) confirm this, and for the sake of brevity we will henceforth ignore terms of the solutions which do not comply to these requirements.

In this section we have obtained leading order solutions for all displacement components and pressure increment in terms of the function $u_{1}^{*(0,0)}$, or more specifically, as linear functions of it and its derivatives. Since knowledge of $u_{1}^{*(0,0)}$ completely determines the long wave high frequency motion at leading order, it is usually referred to as the leading order long wave amplitude, this being independent of $\zeta$. However, this function itself can not be determined without resorting to the higher order. 


\subsubsection{Second order problem}

The second order equations of motion are given by

$$
\begin{aligned}
\gamma_{21} u_{1, \zeta \zeta}^{*(2)}+ & \left(\Lambda_{1}^{e}\right)^{2} u_{1}^{*(2)}=-B_{1111} u_{1, \xi_{1} \xi_{1}}^{*(0)}-\gamma_{31} u_{1, \xi_{3} \xi_{3}}^{*(0)} \\
& -\left(B_{1122}+B_{1221}\right) u_{2, \xi_{1} \zeta}^{*(0)}+\gamma_{21} p_{t, \xi_{1}}^{*(0)}+\eta^{-2}\left(\gamma_{21} u_{1, \tau \tau}^{*(0)}+\left(\Lambda_{1}^{e}\right)^{2} u_{1}^{*(0)}\right), \\
B_{2222} u_{2, \zeta \zeta}^{*(2)}+ & \left(\Lambda_{1}^{e}\right)^{2} u_{2}^{*(2)}+\left(B_{1122}+B_{1221}\right) u_{1, \xi_{1} \zeta}^{*(2)}-\gamma_{21} p_{t, \zeta}^{*(2)}=-\gamma_{12} u_{2, \xi_{1} \xi_{1}}^{*(0)} \\
& -\gamma_{32} u_{2, \xi_{3} \xi_{3}}^{*(0)}-\left(B_{2233}+B_{2332}\right) u_{3, \xi_{3} \zeta}^{*(0)}+\eta^{-2}\left(\gamma_{21} u_{2, \tau \tau}^{*(0)}+\left(\Lambda_{1}^{e}\right)^{2} u_{2}^{*(0)}\right),
\end{aligned}
$$

where we remark that the final terms in each case are of the same order as others, see (4.12). We need not consider the third equation of motion (4.6) to derive the third order model, which is the reason why it is omitted here. Equations (4.24) and (4.25) must be solved subject to the second order incompressibility condition

$$
u_{1, \xi_{1}}^{*(2)}+u_{2, \zeta}^{*(2)}=-u_{3, \xi_{3}}^{*(0)}
$$

and boundary conditions

$$
\begin{gathered}
\gamma_{21} u_{1, \zeta}^{*(2)}=-\left(B_{1221}+\bar{p}\right) u_{2, \xi_{1}}^{*(0)} \quad \text { at } \zeta= \pm 1 \\
\left(B_{2222}+\bar{p}\right) u_{2, \zeta}^{*(2)}+B_{1122} u_{1, \xi_{1}}^{*(2)}-\gamma_{21} p_{t}^{*(2)}=-B_{2233} u_{3, \xi_{3}}^{*(0)} \quad \text { at } \zeta= \pm 1 .
\end{gathered}
$$

The first equation of motion (4.24), when examined in conjunction with the leading order solutions (4.20)-(4.23) and subject to the boundary condition (4.27), indicates that

$$
\begin{aligned}
u_{1}^{*(2)}= & u_{1}^{*(2,0)} \cos \left(\frac{\Lambda_{1}^{e} \zeta}{\sqrt{\gamma_{21}}}\right)+U_{1}^{*(2,0)}, \\
& U_{1}^{*(2,0)}=-\frac{2 \gamma_{21}-\sigma_{2}}{\left(\Lambda_{1}^{e}\right)^{2}} \cos \left(\frac{\Lambda_{1}^{e}}{\sqrt{\gamma_{21}}}\right) u_{1, \xi_{1} \xi_{1}}^{*(0,0)},
\end{aligned}
$$

within which the function $u_{1}^{*(2,0)}$ can not be determined without considering the third order problem. The solution (4.29) is only valid provided

$$
\gamma_{21} u_{1, \tau \tau}^{*(0,0)}+\left(\Lambda_{1}^{e}\right)^{2} u_{1}^{*(0,0)}-\eta^{2}\left(\mathcal{E}_{1 c}^{(2)} u_{1, \xi_{1} \xi_{1}}^{*(0,0)}+\mathcal{E}_{1 s}^{(2)} u_{1, \xi_{3} \xi_{3}}^{*(0,0)}\right)=0 .
$$

Since the displacement component $u_{2}^{*(0)}$ is a linear function of $u_{1}^{*(0,0)}$ (and its derivatives), see (4.21), it will also satisfy equation (4.30). This fact may be used to dispose of the term of $O\left(\eta^{2}\right)$ in the second equation of motion (4.25) and determine solutions for $u_{2}^{*(0)}$ and $p_{t}^{*(0)}$, these being given by

$$
\begin{aligned}
u_{2}^{*(2)} & =u_{2}^{*(2,0)} \sin \left(\frac{\Lambda_{1}^{e} \zeta}{\sqrt{\gamma_{21}}}\right)+\zeta U_{2}^{*(2,1)}, \\
\gamma_{21} p_{t}^{*(2)} & =p_{t}^{*(2,0)} \cos \left(\frac{\Lambda_{1}^{e} \zeta}{\sqrt{\gamma_{21}}}\right)+\zeta^{2} P_{t}^{*(2,2)}+P_{t}^{*(2,0)},
\end{aligned}
$$


where

$$
\begin{gathered}
u_{2}^{*(2,0)}=\sqrt{\gamma_{21}}\left(\frac{\gamma_{21}\left(\gamma_{21}+\mu_{13}\right)}{\gamma_{23}-\gamma_{21}} \frac{\left.\left.u_{1, \xi_{1} \xi_{3} \xi_{3}}^{(0,0)}-\frac{u_{1, \xi_{1}}^{*(2,0)}}{\Lambda_{1}^{e}}\right), \quad \Lambda_{1}^{e}\right)^{3}}{\Lambda_{1}^{(2,1)}}=\frac{2 \Gamma_{1}^{e(2)}}{\left(\Lambda_{1}^{e}\right)^{2}},\right. \\
p_{t}^{*(2,0)}=\left(\gamma_{21}-B_{2222}+B_{1122}+B_{1221}\right) u_{1, \xi_{1}}^{*(2,0)}-\frac{\gamma_{21} \mathcal{Q}_{1 c}^{(-2)}}{\left(\Lambda_{1}^{e}\right)^{2}} u_{1, \xi_{1} \xi_{1} \xi_{1}}^{*(0,0)} \\
-\frac{\gamma_{21}}{\left(\Lambda_{1}^{e}\right)^{2}}\left(\frac{\left(\gamma_{21}+\mu_{13}\right)\left(\gamma_{21}-B_{2222}+B_{2233}+B_{2332}\right)}{\gamma_{23}-\gamma_{21}}+\gamma_{31}-\gamma_{32}\right) u_{1, \xi_{1} \xi_{3} \xi_{3}}^{*(0,0)}, \\
P_{t}^{*(2,0)}=\Gamma_{1}^{e(2)}\left(2 \frac{\gamma_{21}-\sigma_{2}+B_{2222}-B_{1221}}{\left(\Lambda_{1}^{e}\right)^{2}}-1\right)-\frac{1}{\left(\Lambda_{1}^{e}\right)^{2}}\left\{\left(\Lambda_{1}^{e}\right)^{2}\left(2 \gamma_{21}-\sigma_{2}\right) u_{1, \xi_{1}}^{*(2,0)}\right. \\
+\left(B_{2233}\left(2 \gamma_{21}-\sigma_{2}\right)-\gamma_{21}\left(\frac{\left(\gamma_{21}+\mu_{13}\right)\left(\gamma_{23}+\gamma_{21}-\sigma_{2}\right)}{\gamma_{23}-\gamma_{21}}+\gamma_{31}-\gamma_{32}\right)\right) u_{1, \xi_{1} \xi_{3} \xi_{3}}^{*(0,0)} \\
\left.+\left(B_{2211}\left(2 \gamma_{21}-\sigma_{2}\right)-\gamma_{21} \mathcal{Q}_{1 c}^{(-2)}\right) u_{1, \xi_{1} \xi_{1} \xi_{1}}^{*(0,0)}\right\} \cos \left(\frac{\Lambda_{1}^{e}}{\sqrt{\gamma_{21}}}\right),
\end{gathered}
$$

and

$$
P_{t}^{*(2,2)}=\Gamma_{1}^{e(2)}=\frac{2 \gamma_{21}-\sigma_{2}}{2}\left(u_{1, \xi_{1} \xi_{1} \xi_{1}}^{*(0,0)}+u_{1, \xi_{1} \xi_{3} \xi_{3}}^{*(0,0)}\right) \cos \left(\frac{\Lambda_{1}^{e}}{\sqrt{\gamma_{21}}}\right) .
$$

We shall now take a closer look at the equation (4.30). Returning back to the original non-scaled variables and introducing $u^{(0)}\left(x_{1}, x_{3}, t\right) \equiv u_{1}^{*(0,0)}\left(\xi_{1}, \xi_{3}, \tau\right)$ yields

$$
\rho h^{2} \frac{\partial^{2} u^{(0)}}{\partial t^{2}}+\left(\Lambda_{1}^{e}\right)^{2} u^{(0)}-h^{2}\left(\mathcal{E}_{1 c}^{(2)} \frac{\partial^{2} u^{(0)}}{\partial x_{1}^{2}}+\mathcal{E}_{1 s}^{(2)} \frac{\partial^{2} u^{(0)}}{\partial x_{3}^{2}}\right)=0,
$$

in which $\mathcal{E}_{1 c}^{(2)}$ and $\mathcal{E}_{1 s}^{(2)}$ are the functions of material parameters and pre-stress defined immediately after expansion (3.9). It was mentioned previously that the solution of the twodimensional equation (4.33), termed the leading order long wave amplitude, will completely determine the extensional long wave high frequency motion of the layer at leading order. Moreover, if this equation is satisfied, then the second order problem will also be satisfied. We also remark that the associated dispersion relation, derived by substituting the solution of the form (2.9) into the equation (4.33), will match the first two orders of the frequency expansion (3.9), effectively demonstrating a high level of consistency of the model.

It is worth noting, that when the coefficients $\mathcal{E}_{1 c}^{(2)}$ and $\mathcal{E}_{1 s}^{(2)}$ are positive, equation (4.33) is hyperbolic. However, it is possible to find stable pre-stressed states for which the coefficient $\mathcal{E}_{1 c}^{(2)}$ becomes negative, note that $(2.12)$ indicates that $\mathcal{E}_{1 s}^{(2)}$ must always be positive. In such a case equation (4.33) still stays hyperbolic, but it is not a proper wave equation, in a sense that time $t$ and third spatial variable $x_{3}$ swap roles. This result complements a previous observation in a plane strain case, for which a similar change of the coefficient sign leads to loss of hyperbolicity, see Kaplunov et al. (2000b). This behaviour is also related to the possible existence of harmonics with negative group velocity at low wave number. 


\subsubsection{Third order problem}

We will not solve the third order problem completely, but merely derive a refined governing equation for the long wave amplitude. Accordingly, we only consider the third order approximation of the first equation of motion, namely

$$
\begin{aligned}
\gamma_{21} u_{1, \zeta \zeta}^{*(4)}+\left(\Lambda_{1}^{e}\right)^{2} u_{1}^{*(4)}=-\left(B_{1133}+B_{1331}\right) u_{3, \xi_{1} \xi_{3}}^{*(0)}-B_{1111} u_{1, \xi_{1} \xi_{1}}^{*(2)}-\gamma_{31} u_{1, \xi_{3} \xi_{3}}^{*(2)} \\
-\left(B_{1122}+B_{1221}\right) u_{2, \xi_{1} \zeta}^{*(2)}+\gamma_{21} p_{t, \xi_{1}}^{*(2)}+\eta^{-2}\left(\gamma_{21} u_{1, \tau \tau}^{*(2)}+\left(\Lambda_{1}^{e}\right)^{2} u_{1}^{*(2)}\right),
\end{aligned}
$$

subject to the appropriate order boundary condition

$$
\gamma_{21} u_{1, \zeta}^{*(4)}+\left(B_{1221}+\bar{p}\right) u_{2, \xi_{1}}^{*(2)}=0 .
$$

Substituting the functions on the right hand side of the equation of motion (4.34) with their solutions (4.23), (4.29), (4.31) and (4.32), which were obtained in previous sections, we observe that the solution for $u_{1}^{*(4)}$ has the general form

$$
u_{1}^{*(4)}=u_{1}^{*(4,0)} \cos \left(\frac{\Lambda_{1}^{e} \zeta}{\sqrt{\gamma_{21}}}\right)+\zeta u_{1}^{*(4,1)} \sin \left(\frac{\Lambda_{1}^{e} \zeta}{\sqrt{\gamma_{21}}}\right)+\zeta^{2} U_{1}^{*(4,2)}+U_{1}^{*(4,0)} .
$$

Utilising the boundary condition (4.35), and the restriction on $u_{1}^{*(0,0)}$ imposed by (4.30), the following solutions for the coefficients of the (4.36) are derived

$$
\begin{gathered}
u_{1}^{*(4,1)}=-2 \frac{2 \gamma_{21}-\sigma_{2}}{\sqrt{\gamma_{21}}\left(\Lambda_{1}^{e}\right)^{3}} \Gamma_{1}^{e(4)}, \quad U_{1}^{*(4,2)}=\frac{\Gamma_{1}^{e(4)}}{\left(\Lambda_{1}^{e}\right)^{2}} \cos \left(\frac{\Lambda_{1}^{e}}{\sqrt{\gamma_{21}}}\right), \\
U_{1}^{*(4,0)}=\frac{1}{\left(\Lambda_{1}^{e}\right)^{4}}\left\{\gamma_{21}\left(2\left(2 \gamma_{21}-\sigma_{2}\right)+\mathcal{Q}_{1 c}^{(-2)}\right) u_{1, \xi_{1} \xi_{1} \xi_{1} \xi_{1}}^{*(0,0)}-\left(2 \sigma_{2}+2 \gamma_{21}+\left(\Lambda_{1}^{e}\right)^{2}\right) \Gamma_{1}^{e(4)}\right. \\
-\left(\Lambda_{1}^{e}\right)^{2}\left(2 \gamma_{21}-\sigma_{2}\right) u_{1, \xi_{1} \xi_{1}}^{*(2,0)}+\left(\gamma_{21}\left(\frac{\left(\gamma_{21}+\mu_{13}\right)\left(\gamma_{23}+\gamma_{21}-\sigma_{2}\right)}{\gamma_{23}-\gamma_{21}}+\gamma_{31}-\gamma_{32}\right)\right. \\
\left.\left.+\left(2 \gamma_{21}-\sigma_{2}\right)\left(\gamma_{23}-\mu_{13}\right)\right) u_{1, \xi_{1} \xi_{1} \xi_{3} \xi_{3}}^{*(0,0)}\right\} \cos \left(\frac{\Lambda_{1}^{e}}{\sqrt{\gamma_{21}}}\right),
\end{gathered}
$$

within which

$$
\Gamma_{1}^{e(4)}=\frac{2 \gamma_{21}-\sigma_{2}}{2}\left(u_{1, \xi_{1} \xi_{1} \xi_{1} \xi_{1}}^{*(0,0)}+u_{1, \xi_{1} \xi_{1} \xi_{3} \xi_{3}}^{*(0,0)}\right) .
$$

The general solution (4.36) will only satisfy the equation of motion (4.34) provided

$$
\begin{aligned}
\gamma_{21} u_{1, \tau \tau}^{*(2,0)}+\left(\Lambda_{1}^{e}\right)^{2} u_{1}^{*(2,0)}-\eta^{2}\left(\mathcal{E}_{1 c}^{(2)} u_{1, \xi_{1} \xi_{1}}^{*(2,0)}+\mathcal{E}_{1 s}^{(2)} u_{1, \xi_{3} \xi_{3}}^{*(2,0)}\right. \\
\left.-\mathcal{E}_{1 c}^{(4)} u_{1, \xi_{1} \xi_{1} \xi_{1} \xi_{1}}^{*(0,0)}-\mathcal{E}_{1 s}^{(4)} u_{1, \xi_{1} \xi_{1} \xi_{3} \xi_{3}}^{*(0,0)}\right)=0,
\end{aligned}
$$

this may be used together with equation (4.30) to refine the leading order long wave amplitude $u^{*(0)}$. To do this we multiply equation (4.37) by $\eta^{2}$ and add the result to equation (4.30), which upon the introduction of the non-scaled and scaled long wave amplitude expansions

$$
u=u^{(0)}+u^{(2)} \eta^{2}+O\left(\eta^{4}\right), \quad u^{*}=u^{*(0)}+u^{*(2)} \eta^{2}+O\left(\eta^{4}\right),
$$


gives the following governing equation

$$
\begin{aligned}
\gamma_{21} u_{, \tau \tau}^{*}+\left(\Lambda_{1}^{e}\right)^{2} u^{*}-\eta^{2}\left(\mathcal{E}_{1 c}^{(2)} u_{, \xi_{1} \xi_{1}}^{*}+\mathcal{E}_{1 s}^{(2)} u_{, \xi_{3} \xi_{3}}^{*}\right) & \\
+ & \eta^{4}\left(\mathcal{E}_{1 c}^{(4)} u_{, \xi_{1} \xi_{1} \xi_{1} \xi_{1}}^{*}+\mathcal{E}_{1 s}^{(4)} u_{, \xi_{1} \xi_{1} \xi_{3} \xi_{3}}^{*}\right)=0,
\end{aligned}
$$

where notation introduced immediately after (3.9) has been utilised. This equation can be expressed in terms of the original (non-scaled) variables, giving

$$
\rho h^{2} \frac{\partial^{2} u}{\partial t^{2}}+\left(\Lambda_{1}^{e}\right)^{2} u-h^{2}\left(\mathcal{E}_{1 c}^{(2)} \frac{\partial^{2} u}{\partial x_{1}^{2}}+\mathcal{E}_{1 s}^{(2)} \frac{\partial^{2} u}{\partial x_{3}^{2}}\right)+h^{4}\left(\mathcal{E}_{1 c}^{(4)} \frac{\partial^{4} u}{\partial x_{1}^{4}}+\mathcal{E}_{1 s}^{(4)} \frac{\partial^{4} u}{\partial x_{1}^{2} \partial x_{3}^{2}}\right)=0 .
$$

When this is satisfied, all the equations of third order problem will also be satisfied. It is easy to see from our solutions, that each displacement component and the pressure increment are represented as linear functions of $u_{1}^{*(0,0)}$ and $u_{1}^{*(2,0)}$. Moreover, the functions $u_{1}^{*(2 m, 0)}, m=0,1,2 \ldots$ will all satisfy each of the boundary value problems posed for $u_{1}^{*(0,0)}, \ldots u_{1}^{*(2 m-2,0)}$, indicating that the displacement components and pressure increment are linear functions of the long wave amplitude and its derivatives. We may, as we did it for the second order governing equation for the long wave amplitude (4.33), substitute the solution of the form (2.9) into the (4.40) and derive the corresponding dispersion relation. The refined result will match our expansion for the scaled frequency expansion (3.9) exactly.

\subsubsection{Higher order approximations}

For certain problems it may be useful to derive even higher order approximations. Generally, the equations of motion of fourth and higher order $(m \geqslant 2)$ are given by

$$
\begin{aligned}
& \gamma_{21} u_{1, \zeta \zeta}^{*(2 m+2)}+\left(\Lambda_{1}^{e}\right)^{2} u_{1}^{*(2 m+2)}=-B_{1111} u_{1, \xi_{1} \xi_{1}}^{*(2 m)}-\gamma_{31} u_{1, \xi_{3} \xi_{3}}^{*(2 m)}-\left(B_{1122}+B_{1221}\right) u_{2, \xi_{1} \zeta}^{*(2 m)} \\
& \quad+\gamma_{21} p_{t, \xi_{1}}^{*(2 m)}+\eta^{-2}\left(\gamma_{21} u_{1, \tau \tau}^{*(2 m)}+\left(\Lambda_{1}^{e}\right)^{2} u_{1}^{*(2 m)}\right)-\left(B_{1133}+B_{1331}\right) u_{3, \xi_{1} \xi_{3}}^{*(2 m-2)} \\
& B_{2222} u_{2, \zeta \zeta}^{*(2 m+2)}+\left(\Lambda_{1}^{e}\right)^{2} u_{2}^{*(2 m+2)}+\left(B_{1122}+B_{1221}\right) u_{1, \xi_{1} \zeta}^{*(2 m+2)}-\gamma_{21} p_{t, \zeta}^{*(2 m+2)}=-\gamma_{12} u_{2, \xi_{1} \xi_{1}}^{*(2 m)} \\
& \quad-\gamma_{32} u_{2, \xi_{3} \xi_{3}}^{*(2 m)}-\left(B_{2233}+B_{2332}\right) u_{3, \xi_{3} \zeta}^{*(2 m)}+\eta^{-2}\left(\gamma_{21} u_{2, \tau \tau}^{*(2 m)}+\left(\Lambda_{1}^{e}\right)^{2} u_{2}^{*(2 m)}\right) \\
& \gamma_{23} u_{3, \zeta \zeta}^{*(2 m+2)}+\left(\Lambda_{1}^{e}\right)^{2} u_{3}^{*(2 m+2)}+\left(B_{1133}+B_{1331}\right) u_{1, \xi_{1} \xi_{3}}^{*(2 m+2)}+\left(B_{2233}+B_{2332}\right) u_{2, \zeta \xi_{3}}^{*(2 m+2)} \\
& \quad-\gamma_{21} p_{t, \xi_{3}}^{*(2 m+2)}=-\gamma_{13} u_{3, \xi_{1} \xi_{1}}^{*(2 m)}-B_{3333} u_{3, \xi_{3} \xi_{3}}^{*(2 m)}+\eta^{-2}\left(\gamma_{21} u_{3, \tau \tau}^{*(2 m)}+\left(\Lambda_{1}^{e}\right)^{2} u_{3}^{*(2 m)}\right)
\end{aligned}
$$

these must be solved in conjunction with the incompressibility condition

$$
u_{1, \xi_{1}}^{*(2 m+2)}+u_{2, \zeta}^{*(2 m+2)}=-u_{3, \xi_{3}}^{*(2 m)},
$$

and subject to the boundary conditions

$$
\begin{gathered}
\gamma_{21} u_{1, \zeta}^{*(2 m+2)}=-\left(B_{1221}+\bar{p}\right) u_{2, \xi_{1}}^{*(2 m)} \quad \text { at } \zeta= \pm 1 \\
\left(B_{2222}+\bar{p}\right) u_{2, \zeta}^{*(2 m+2)}+B_{1122} u_{1, \xi_{1}}^{*(2 m+2)}-\gamma_{21} p_{t}^{*(2 m+2)}=-B_{2233} u_{3, \xi_{3}}^{*(2 m)} \quad \text { at } \zeta= \pm 1, \\
\gamma_{23} u_{3, \zeta}^{*(2 m+2)}+\left(B_{2332}+\bar{p}\right) u_{2, \xi_{3}}^{*(2 m+2)}=0 \quad \text { at } \zeta= \pm 1 .
\end{gathered}
$$


It is possible to specify the general solution for this problem, so that the process of solution will simplify to a system of algebraic equations, the general solutions of which may be represented in the following form

$$
\begin{gathered}
u_{i}^{*(2 m+2)=} \sum_{n=0}^{m+1} u_{i}^{*(2 m+2, n)} \zeta^{n} \cos \left(\frac{\pi n}{2}+\frac{\Lambda_{1}^{e} \zeta}{\sqrt{\gamma_{21}}}\right)+\sum_{n=0}^{m+1} U_{i}^{*(2 m+2,2 n)} \zeta^{2 n} \\
\quad+\sum_{n=0}^{m} v_{i}^{*(2 m+2, n)} \zeta^{n} \cos \left(\frac{\pi n}{2}+\frac{\Lambda_{1}^{e} \zeta}{\sqrt{\gamma_{23}}}\right), \quad i \in\{1,3\}, \\
u_{2}^{*(2 m+2)}=\sum_{n=0}^{m+1} u_{2}^{*(2 m+2, n)} \zeta^{n} \sin \left(\frac{\pi n}{2}+\frac{\Lambda_{1}^{e} \zeta}{\sqrt{\gamma_{21}}}\right) \\
+\sum_{n=0}^{m} U_{2}^{*(2 m+2,2 n+1)} \zeta^{2 n+1}+\sum_{n=0}^{m} v_{2}^{*(2 m+2, n)} \zeta^{n} \sin \left(\frac{\pi n}{2}+\frac{\Lambda_{1}^{e} \zeta}{\sqrt{\gamma_{23}}}\right), \\
p_{t}^{*(2 m+2)}=\sum_{n=0}^{m+1} p_{t}^{*(2 m+2, n)} \zeta^{n} \cos \left(\frac{\pi n}{2}+\frac{\Lambda_{1}^{e} \zeta}{\sqrt{\gamma_{21}}}\right) \\
\quad+\sum_{n=0}^{m+1} P_{t}^{*(2 m+2,2 n)} \zeta^{2 n}+\sum_{n=0}^{m} \tilde{p}_{t}^{*(2 m+2, n)} \zeta^{n} \cos \left(\frac{\pi n}{2}+\frac{\Lambda_{1}^{e} \zeta}{\sqrt{\gamma_{23}}}\right),
\end{gathered}
$$

in which arguments of sines and cosines are chosen to ensure that the solutions will have appropriate symmetric or anti-symmetric structure. Though possible, the solving of the fourth or higher order problem will face severe algebraic difficulties. However, with the advent of modern computer algebraic manipulation packages such a method is possible and such problems are mathematically tractable.

\subsection{Second family of the cut-off frequencies}

It follows from the approximations (3.11), that for the motions in the vicinity of second family of cut-off frequencies a typical wave travels the distance of a typical wave length $l$ in a time $t \sim l \frac{\eta \sqrt{\rho}}{\Lambda_{3}^{e}}$, so the suitable rescaling of time is

$$
t=l \eta \sqrt{\frac{\rho}{\gamma_{23}}} \tau .
$$

Motivated by the estimations (3.17) we rescale the displacements and pressure so that

$$
\begin{gathered}
u_{m}\left(x_{1}, x_{2}, x_{3}, t\right)=l \eta^{3-m} u_{m}^{*}\left(\xi_{1}, \zeta, \xi_{3}, \tau\right), \quad m \in\{1,2,3\}, \\
p_{t}\left(x_{1}, x_{2}, x_{3}, t\right)=\gamma_{23} p_{t}^{*}\left(\xi_{1}, \zeta, \xi_{3}, \tau\right) .
\end{gathered}
$$


When appropriately rescaled, the equations of motions (2.3)-(2.5) are given by

$$
\begin{gathered}
\gamma_{21} u_{1, \zeta \zeta}^{*}+\left(\Lambda_{3}^{e}\right)^{2} u_{1}^{*}-\left\{\left(\Lambda_{3}^{e}\right)^{2} u_{1}^{*}+\gamma_{23} u_{1, \tau \tau}^{*}\right\}+\left(B_{1122}+B_{1221}\right) u_{2, \xi_{1} \zeta}^{*} \\
+\left(B_{1133}+B_{1331}\right) u_{3, \xi_{1} \xi_{3}}^{*}-\gamma_{23} p_{t, \xi_{1}}^{*}+\eta^{2}\left(B_{1111} u_{1, \xi_{1} \xi_{1}}^{*}+\gamma_{31} u_{1, \xi_{3} \xi_{3}}^{*}\right)=0, \\
B_{2222} u_{2, \zeta \zeta}^{*}+\left(\Lambda_{3}^{e}\right)^{2} u_{2}^{*}-\left\{\left(\Lambda_{3}^{e}\right)^{2} u_{2}^{*}+\gamma_{23} u_{2, \tau \tau}^{*}\right\}+\left(B_{2233}+B_{2332}\right) u_{3, \xi_{3} \zeta}^{*} \\
-\gamma_{23} p_{t, \zeta}^{*}+\eta^{2}\left(\left(B_{1122}+B_{1221}\right) u_{1, \xi_{1} \zeta}^{*}+\gamma_{12} u_{2, \xi_{1} \xi_{1}}^{*}+\gamma_{32} u_{2, \xi_{3} \xi_{3}}^{*}\right)=0 \\
\gamma_{23} u_{3, \zeta \zeta}^{*}+\left(\Lambda_{3}^{e}\right)^{2} u_{3}^{*}-\left\{\left(\Lambda_{3}^{e}\right)^{2} u_{3}^{*}+\gamma_{23} u_{3, \tau \tau}^{*}\right\}+\eta^{2}\left(\left(B_{2233}+B_{2332}\right) u_{2, \xi_{3} \zeta}^{*}\right. \\
\left.+\gamma_{13} u_{3, \xi_{1} \xi_{1}}^{*}+B_{3333} u_{3, \xi_{3} \xi_{3}}^{*}-\gamma_{21} p_{t, \xi_{3}}^{*}\right)+\eta^{4}\left(B_{1133}+B_{1331}\right) u_{1, \xi_{1} \xi_{3}}^{*}=0
\end{gathered}
$$

these must be solved together with the incompressibility condition

$$
u_{2, \zeta}^{*}+u_{3, \xi_{3}}^{*}+\eta^{2} u_{1, \xi_{1}}^{*}=0
$$

subject to the boundary conditions

$$
\begin{gathered}
\gamma_{21} u_{1, \zeta}^{*}+\left(B_{1221}+\bar{p}\right) u_{2, \xi_{1}}^{*}=0 \quad \text { at } \zeta= \pm 1, \\
\left(B_{2222}+\bar{p}\right) u_{2, \zeta}^{*}+B_{2233} u_{3, \xi_{3}}^{*}-\gamma_{21} p_{t}^{*}+\eta^{2} B_{1122} u_{1, \xi_{1}}^{*}=0 \quad \text { at } \zeta= \pm 1, \\
\gamma_{23} u_{3, \zeta}^{*}+\eta^{2}\left(B_{2332}+\bar{p}\right) u_{2, \xi_{3}}^{*}=0 \quad \text { at } \zeta= \pm 1 .
\end{gathered}
$$

As we did in the previous case, we may utilise the fact that

$$
\gamma_{23} u_{m, \tau \tau}^{*}+\left(\Lambda_{3}^{e}\right)^{2} u_{m}^{*} \sim \eta^{2} u_{m}^{*}, \quad m \in\{1,2,3\},
$$

to reconsider the order of terms in the braces in the equations of motion (4.53)-(4.53).

\subsubsection{Leading order problem}

For the leading order problem we are picking up the leading order terms of the equations (4.53)-(4.59), yielding the equations of motion

$$
\begin{aligned}
& \gamma_{21} u_{1, \zeta \zeta}^{*(0)}+\left(\Lambda_{3}^{e}\right)^{2} u_{1}^{*(0)}+\left(B_{1122}+B_{1221}\right) u_{2, \xi_{1} \zeta}^{*(0)} \\
&+\left(B_{1133}+B_{1331}\right) u_{3, \xi_{1} \xi_{3}}^{*(0)}-\gamma_{23} p_{t, \xi_{1}}^{*(0)}=0, \\
& B_{2222} u_{2, \zeta \zeta}^{*(0)}+\left(\Lambda_{3}^{e}\right)^{2} u_{2}^{*(0)}+\left(B_{2233}+B_{2332}\right) u_{3, \xi_{3} \zeta}^{*(0)}-\gamma_{23} p_{t, \zeta}^{*(0)}=0 \\
& \gamma_{23} u_{3, \zeta \zeta}^{*(0)}+\left(\Lambda_{3}^{e}\right)^{2} u_{3}^{*(0)}=0,
\end{aligned}
$$

and the leading order incompressibility condition

$$
u_{2, \zeta}^{*(0)}+u_{3, \xi_{3}}^{*(0)}=0 .
$$


Equations (4.61)-(4.64) must be solved in conjunction with the leading order boundary conditions, given by

$$
\begin{gathered}
\gamma_{21} u_{1, \zeta}^{*(0)}+\left(B_{1221}+\bar{p}\right) u_{2, \xi_{1}}^{*(0)}=0 \quad \text { at } \zeta= \pm 1, \\
\left(B_{2222}+\bar{p}\right) u_{2, \zeta}^{*(0)}+B_{2233} u_{3, \xi_{3}}^{*(0)}-\gamma_{23} p_{t}^{*(0)}=0 \quad \text { at } \zeta= \pm 1, \\
\gamma_{23} u_{3, \zeta}^{*(0)}=0 \quad \text { at } \zeta= \pm 1 .
\end{gathered}
$$

The solution for the system (4.61)-(4.67) is readily established in the following form

$$
\begin{array}{ll}
u_{1}^{*(0)}=u_{1}^{*(0,0)} \cos \left(\frac{\Lambda_{3}^{e} \zeta}{\sqrt{\gamma_{23}}}\right)+U_{1}^{*(0,0)}, & u_{2}^{*(0)}=u_{2}^{*(0,0)} \sin \left(\frac{\Lambda_{3}^{e} \zeta}{\sqrt{\gamma_{23}}}\right), \\
u_{3}^{*(0)}=u_{3}^{*(0,0)} \cos \left(\frac{\Lambda_{3}^{e} \zeta}{\sqrt{\gamma_{23}}}\right), & \gamma_{21} p_{t}^{*(0)}=p_{t}^{*(0,0)} \cos \left(\frac{\Lambda_{3}^{e} \zeta}{\sqrt{\gamma_{23}}}\right)+P_{t}^{*(0,0)},
\end{array}
$$

in which

$$
\begin{aligned}
& u_{1}^{*(0,0)}=\frac{\gamma_{23}\left(\gamma_{23}+\mu_{13}\right)}{\left(\gamma_{23}-\gamma_{21}\right)\left(\Lambda_{3}^{e}\right)^{2}} u_{3, \xi_{1} \xi_{3}}^{*(0,0)}, \quad U_{1}^{*(0,0)}=-\frac{2 \gamma_{23}-\sigma_{2}}{\left(\Lambda_{3}^{e}\right)^{2}} \cos \left(\frac{\Lambda_{3}^{e}}{\sqrt{\gamma_{23}}}\right) u_{3, \xi_{1} \xi_{3}}^{*(0,0)}, \\
& u_{2}^{*(0,0)}=-\frac{\sqrt{\gamma_{23}}}{\Lambda_{3}^{e}} u_{3, \xi_{3}}^{*(0,0)}, \quad P_{t}^{*(0,0)}=-\left(2 \gamma_{23}-\sigma_{2}\right) u_{3, \xi_{3}}^{*(0,0)} \cos \left(\frac{\Lambda_{3}^{e}}{\sqrt{\gamma_{23}}}\right) \\
& p_{t}^{*(0,0)}=\left(\gamma_{23}-B_{2222}+B_{2233}+B_{2332}\right) u_{3, \xi_{3}}^{*(0,0)} .
\end{aligned}
$$

To determine the function $u_{3}^{*(0,0)}$ we have to resort to higher order. For the considered case $u_{3}^{*(0,0)}$ plays role of the leading order long wave amplitude, so every function in (4.68) is the linear function of $u_{3}^{*(0,0)}$ and its derivatives.

\subsubsection{Second order problem}

In order to derive the leading order governing equation for the long wave amplitude, and its correction at third order, we need only consider the appropriate second and third equations of motions

$$
\begin{aligned}
B_{2222} u_{2, \zeta \zeta}^{*(2)}+ & \left(\Lambda_{3}^{e}\right)^{2} u_{2}^{*(2)}-\left(B_{2233}+B_{2332}\right) u_{3, \xi_{3} \zeta}^{*(2)}-\gamma_{23} p_{t, \zeta}^{*(2)}=-\gamma_{12} u_{2, \xi_{1} \xi_{1}}^{*(0)} \\
& -\gamma_{32} u_{2, \xi_{3} \xi_{3}}^{*(0)}-\left(B_{1122}+B_{1221}\right) u_{1, \xi_{1} \zeta}^{*(0)}+\eta^{-2}\left(\gamma_{23} u_{2, \tau \tau}^{*(0)}+\left(\Lambda_{3}^{e}\right)^{2} u_{2}^{*(0)}\right) \\
\gamma_{23} u_{3, \zeta \zeta}^{*(2)}+ & \left(\Lambda_{3}^{e}\right)^{2} u_{3}^{*(2)}=-\gamma_{13} u_{3, \xi_{1} \xi_{1}}^{*(0)}-B_{3333} u_{3, \xi_{3} \xi_{3}}^{*(0)} \\
& -\left(B_{2233}+B_{2332}\right) u_{2, \xi_{3} \zeta}^{*(0)}+\gamma_{23} p_{t, \xi_{3}}^{*(0)}+\eta^{-2}\left(\gamma_{23} u_{3, \tau \tau}^{*(0)}+\left(\Lambda_{3}^{e}\right)^{2} u_{3}^{*(0)}\right)
\end{aligned}
$$

and incompressibility condition

$$
u_{2, \zeta}^{*(2)}+u_{3, \xi_{3}}^{*(2)}=-u_{1, \xi_{1}}^{*(0)},
$$

which are to be considered in conjunction with the second and third second order boundary conditions

$$
\begin{gathered}
\left(B_{2222}+\bar{p}\right) u_{2, \zeta}^{*(2)}+B_{2233} u_{3, \xi_{3}}^{*(2)}-\gamma_{23} p_{t}^{*(2)}=-B_{1122} u_{1, \xi_{1}}^{*(0)} \quad \text { at } \zeta= \pm 1 \\
\gamma_{23} u_{3, \zeta}^{*(2)}=-\left(B_{2332}+\bar{p}\right) u_{2, \xi_{3}}^{*(0)} \quad \text { at } \zeta= \pm 1
\end{gathered}
$$


The solution for the system (4.69)-(4.73) is given by

$$
\begin{aligned}
u_{2}^{*(2)} & =u_{2}^{*(2,0)} \sin \left(\frac{\Lambda_{3}^{e} \zeta}{\sqrt{\gamma_{23}}}\right)+\zeta U_{2}^{*(2,1)}, \\
u_{3}^{*(2)} & =u_{3}^{*(2,0)} \cos \left(\frac{\Lambda_{3}^{e} \zeta}{\sqrt{\gamma_{23}}}\right)+U_{3}^{*(2,0)}, \\
\gamma_{23} p_{t}^{*(2)} & =p_{t}^{*(2,0)} \cos \left(\frac{\Lambda_{3}^{e} \zeta}{\sqrt{\gamma_{23}}}\right)+\zeta^{2} P_{t}^{*(2,2)}+P_{t}^{*(2,0)},
\end{aligned}
$$

this solution only being valid provided

$$
\gamma_{23} u_{3, \tau \tau}^{*(0,0)}+\left(\Lambda_{3}^{e}\right)^{2} u_{3}^{*(0,0)}-\eta^{2}\left(\mathcal{E}_{3 c}^{(2)} u_{3, \xi_{1} \xi_{1}}^{*(0,0)}+\mathcal{E}_{3 s}^{(2)} u_{3, \xi_{3} \xi_{3}}^{*(0,0)}\right)=0
$$

Substituting the solutions (4.74) into the equations (4.69)-(4.73) the following representations can be derived

$$
\begin{gathered}
u_{2}^{*(2,0)}=\sqrt{\gamma_{23}}\left(\frac{\gamma_{23}\left(\gamma_{23}+\mu_{13}\right)}{\gamma_{23}-\gamma_{21}} \frac{u_{3, \xi_{1} \xi_{1} \xi_{3}}^{*(0,0)}}{\left(\Lambda_{3}^{e}\right)^{3}}-\frac{u_{3, \xi_{3}}^{*(2,0)}}{\Lambda_{3}^{e}}\right), \\
U_{2}^{*(2,1)}=\frac{2 \Gamma_{3}^{e(2)}}{\left(\Lambda_{3}^{e}\right)^{2}}, \quad U_{3}^{*(2,0)}=-\frac{2 \gamma_{23}-\sigma_{2}}{\left(\Lambda_{3}^{e}\right)^{2}} \cos \left(\frac{\Lambda_{3}^{e}}{\sqrt{\gamma_{23}}}\right) u_{3, \xi_{3} \xi_{3}}^{*(0,0)}, \\
p_{t}^{*(2,0)}=\left(\gamma_{23}-B_{2222}+B_{2233}+B_{2332}\right) u_{3, \xi_{3}}^{*(2,0)}-\frac{\gamma_{23} \mathcal{Q}_{3 s}^{(-2)}}{\left(\Lambda_{3}^{e}\right)^{2}} u_{3, \xi_{3} \xi_{3} \xi_{3}}^{*(0,0)} \\
-\frac{\gamma_{23}}{\left(\Lambda_{3}^{e}\right)^{2}}\left(\frac{\left(\gamma_{23}+\mu_{13}\right)\left(\gamma_{23}-B_{2222}+B_{1122}+B_{1221}\right)}{\left(\Lambda_{12}^{e}\right)^{2}}+\gamma_{12}-\gamma_{13}\right) u_{3, \xi_{1} \xi_{1} \xi_{3}}^{*}, \\
P_{t}^{*(2,0)}=\Gamma_{3}^{e(2)}\left(2 \frac{\gamma_{23}-\sigma_{2}+B_{222}-B_{2332}}{\gamma_{23}}-\frac{1}{\left(\Lambda_{3}^{e}\right)^{2}}\left\{\left(\Lambda_{3}^{e}\right)^{2}\left(2 \gamma_{23}-\sigma_{2}\right) u_{3, \xi_{3}}^{*(2,0)}\right.\right. \\
+\left(B_{1122}\left(2 \gamma_{23}-\sigma_{2}\right)+\gamma_{23}\left(\frac{\left(\gamma_{23}+\mu_{13}\right)\left(\gamma_{23}+\gamma_{21}-\sigma_{2}\right)}{\gamma_{23}-\gamma_{21}}+\gamma_{12}-\gamma_{13}\right)\right) u_{3, \xi_{1} \xi_{1} \xi_{3}}^{*(0,0)} \\
+\left(B_{2233}\left(2 \gamma_{23}-\sigma_{2}\right)-\gamma_{23} \mathcal{Q}_{3 s}^{(-2)}\right) u_{3, \xi_{3} \xi_{3} \xi_{3}}^{*(0,0)} \cos \left(\frac{\Lambda_{3}^{e}}{\sqrt{\gamma_{23}}}\right)
\end{gathered}
$$

and also

$$
P_{t}^{*(2,2)}=\Gamma_{3}^{e(2)}=\frac{2 \gamma_{23}-\sigma_{2}}{2}\left(u_{3, \xi_{1} \xi_{1} \xi_{3}}^{*(0,0)}+u_{3, \xi_{3} \xi_{3} \xi_{3}}^{*(0,0)}\right) \cos \left(\frac{\Lambda_{3}^{e}}{\sqrt{\gamma_{23}}}\right)
$$

This derivation again utilises the fact, that every displacement component $u_{m}^{*(0)}(m \in\{1,3\})$ is a linear function of the leading order long wave amplitude $u_{3}^{*(0)}$ and its derivatives, which guarantees that the equation (4.75) is also valid for each of the displacement components. Rescaling (4.75) back to the original variables we obtain the governing equation for the leading order long wave amplitude

$$
\rho h^{2} \frac{\partial^{2} u^{(0)}}{\partial t^{2}}+\left(\Lambda_{3}^{e}\right)^{2} u^{(0)}-h^{2}\left(\mathcal{E}_{3 c}^{(2)} \frac{\partial^{2} u^{(0)}}{\partial x_{1}^{2}}+\mathcal{E}_{3 s}^{(2)} \frac{\partial^{2} u^{(0)}}{\partial x_{3}^{2}}\right)=0 .
$$

It is again remarked that the dispersion relation associated with this equation matches first two orders of the expansion (3.13). 


\subsubsection{Third order problem}

At third order the third equation of motion is given by

$$
\begin{aligned}
\gamma_{23} u_{3, \zeta \zeta}^{*(4)}+\left(\Lambda_{3}^{e}\right)^{2} u_{3}^{*(4)}=-\left(B_{1133}+B_{1331}\right) u_{1, \xi_{1} \xi_{3}}^{*(0)}-\left(B_{2233}+B_{2332}\right) u_{2, \xi_{3} \zeta}^{*(2)} \\
\quad-\gamma_{13} u_{3, \xi_{1} \xi_{1}}^{*(2)}-B_{3333} u_{3, \xi_{3} \xi_{3}}^{*(2)}+\gamma_{23} p_{t, \xi_{3}}^{*(2)}+\eta^{-2}\left(\gamma_{23} u_{3, \tau \tau}^{*(2)}+\left(\Lambda_{3}^{e}\right)^{2} u_{3}^{*(2)}\right),
\end{aligned}
$$

with the associated boundary condition

$$
\left(B_{2332}+\bar{p}\right) u_{2, \xi_{3}}^{*(2)}+\gamma_{23} u_{3, \zeta}^{*(4)}=0
$$

The problem posed by (4.77) and (4.78) is all that is needed to derive a corrected governing equation for the long wave amplitude. Inserting solutions derived at the previous orders into the right hand side of the equation (4.77) we observe the following form of general solution

$$
u_{3}^{*(4)}=u_{3}^{*(4,0)} \cos \left(\frac{\Lambda_{3}^{e} \zeta}{\sqrt{\gamma_{23}}}\right)+\zeta u_{3}^{*(4,1)} \sin \left(\frac{\Lambda_{3}^{e} \zeta}{\sqrt{\gamma_{23}}}\right)+\zeta^{2} U_{3}^{*(4,2)}+U_{3}^{*(4,0)},
$$

which together with the boundary condition (4.78) yields

$$
\begin{gathered}
u_{3}^{*(4,1)}=-2 \frac{2 \gamma_{23}-\sigma_{2}}{\sqrt{\gamma_{23}}\left(\Lambda_{3}^{e}\right)^{3}} \Gamma_{3}^{e(4)}, \quad U_{3}^{*(4,2)}=\frac{\Gamma_{3}^{e(4)}}{\left(\Lambda_{3}^{e}\right)^{2}} \cos \left(\frac{\Lambda_{3}^{e}}{\sqrt{\gamma_{23}}}\right), \\
U_{3}^{*(4,0)}=\frac{1}{\left(\Lambda_{3}^{e}\right)^{4}}\left\{\gamma_{23}\left(2\left(2 \gamma_{21}-\sigma_{2}\right)+\mathcal{Q}_{3 s}^{(-2)}\right) u_{3, \xi_{3} \xi_{3} \xi_{3} \xi_{3}}^{*(0,0)}-\left(2 \sigma_{2}+2 \gamma_{23}+\left(\Lambda_{3}^{e}\right)^{2}\right) \Gamma_{3}^{e(4)}\right. \\
-\left(\Lambda_{3}^{e}\right)^{2}\left(2 \gamma_{23}-\sigma_{2}\right) u_{3, \xi_{3} \xi_{3}}^{*(2,0)}-\left(\gamma_{23}\left(\frac{\left(\gamma_{23}+\mu_{13}\right)\left(\gamma_{23}+\gamma_{21}-\sigma_{2}\right)}{\gamma_{23}-\gamma_{21}}+\gamma_{12}-\gamma_{13}\right)\right. \\
\left.\left.-\left(2 \gamma_{23}-\sigma_{2}\right)\left(\gamma_{21}-\mu_{13}\right)\right) u_{3, \xi_{1} \xi_{1} \xi_{3} \xi_{3}}^{*(0,0)}\right\} \cos \left(\frac{\Lambda_{3}^{e}}{\sqrt{\gamma_{23}}}\right),
\end{gathered}
$$

where

$$
\Gamma_{3}^{e(4)}=\frac{2 \gamma_{23}-\sigma_{2}}{2}\left(u_{3, \xi_{1} \xi_{1} \xi_{3} \xi_{3}}^{*(0,0)}+u_{3, \xi_{3} \xi_{3} \xi_{3} \xi_{3}}^{*(0,0)}\right) .
$$

Equation (4.77) may only be satisfied provided

$$
\begin{aligned}
\gamma_{23} u_{3, \tau \tau}^{*(2,0)}+\left(\Lambda_{3}^{e}\right)^{2} u_{3}^{*(2,0)}-\eta^{2}\left(\mathcal{E}_{3 c}^{(2)} u_{3, \xi_{1} \xi_{1}}^{*(2,0)}+\mathcal{E}_{3 s}^{(2)} u_{3, \xi_{3} \xi_{3}}^{*(2,0)}\right. & \\
& \left.-\mathcal{E}_{3 c}^{(4)} u_{3, \xi_{1} \xi_{1} \xi_{3} \xi_{3}}^{*(0,0)}-\mathcal{E}_{3 s}^{(4)} u_{3, \xi_{3} \xi_{3} \xi_{3} \xi_{3}}^{*(0,0)}\right)=0,
\end{aligned}
$$

which upon multiplying onto $\eta^{2}$, adding to the (4.75) and rescaling to the original variables gives the governing equation for the long wave amplitude

$$
\rho h^{2} \frac{\partial^{2} u}{\partial t^{2}}+\left(\Lambda_{3}^{e}\right)^{2} u-h^{2}\left(\mathcal{E}_{3 c}^{(2)} \frac{\partial^{2} u}{\partial x_{1}^{2}}+\mathcal{E}_{3 s}^{(2)} \frac{\partial^{2} u}{\partial x_{3}^{2}}\right)+h^{4}\left(\mathcal{E}_{3 c}^{(4)} \frac{\partial^{4} u}{\partial x_{1}^{2} \partial x_{3}^{2}}+\mathcal{E}_{3 s}^{(4)} \frac{\partial^{4} u}{\partial x_{3}^{4}}\right)=0,
$$

in which $u$ is defined by (4.38). 


\subsubsection{Higher order approximations}

Once again, we will only provide the necessary information for deriving higher order approximate equations for the second family of the cut-off frequencies for completeness. The equations of motion for fourth or higher order $(m \geqslant 2)$ are generally given by

$$
\begin{aligned}
& \gamma_{21} u_{1, \zeta \zeta}^{*(2 m+2)}+\left(\Lambda_{3}^{e}\right)^{2} u_{1}^{*(2 m+2)}+\left(B_{1122}+B_{1221}\right) u_{2, \zeta \xi_{1}}^{*(2 m+2)}+\left(B_{1133}+B_{1331}\right) u_{3, \xi_{3} \xi_{1}}^{*(2 m+2)} \\
& -\gamma_{23} p_{t, \xi_{1}}^{*(2 m+2)}=-B_{1111} u_{1, \xi_{1} \xi_{1}}^{*(2 m)}-\gamma_{31} u_{1, \xi_{3} \xi_{3}}^{*(2 m)}+\eta^{-2}\left(\gamma_{23} u_{1, \tau \tau}^{*(2 m)}+\left(\Lambda_{3}^{e}\right)^{2} u_{1}^{*(2 m)}\right) \\
& B_{2222} u_{2, \zeta \zeta}^{*(2 m+2)}+\left(\Lambda_{3}^{e}\right)^{2} u_{2}^{*(2 m+2)}-\left(B_{2233}+B_{2332}\right) u_{3, \xi_{3} \zeta}^{*(2 m+2)}-\gamma_{23} p_{t, \zeta}^{*(2 m+2)}=-\gamma_{12} u_{2, \xi_{1} \xi_{1}}^{*(2 m)} \\
& \quad-\gamma_{32} u_{2, \xi_{3} \xi_{3}}^{*(2 m)}-\left(B_{1122}+B_{1221}\right) u_{1, \xi_{1} \zeta}^{*(2 m)}+\eta^{-2}\left(\gamma_{23} u_{2, \tau \tau}^{*(2 m)}+\left(\Lambda_{3}^{e}\right)^{2} u_{2}^{*(2 m)}\right) \\
& \gamma_{23} u_{3, \zeta \zeta}^{*(2 m+2)}+\left(\Lambda_{3}^{e}\right)^{2} u_{3}^{*(2 m+2)}=-\left(B_{2233}+B_{2332}\right) u_{2, \xi_{3} \zeta}^{*(2 m)}-\gamma_{13} u_{3, \xi_{1} \xi_{1}}^{*(2 m)}-B_{3333} u_{3, \xi_{3} \xi_{3}}^{*(2 m)} \\
& \quad+\gamma_{23} p_{t, \xi_{3}}^{*(2 m)}+\eta^{-2}\left(\gamma_{23} u_{3, \tau \tau}^{*(2 m)}+\left(\Lambda_{3}^{e}\right)^{2} u_{3}^{*(2 m)}\right)-\left(B_{1133}+B_{1331}\right) u_{1, \xi_{1} \xi_{3}}^{*(2 m-2)}
\end{aligned}
$$

and must be solved in conjunction with the incompressibility condition

$$
u_{2, \zeta}^{*(2 m+2)}+u_{3, \xi_{3}}^{*(2 m+2)}=-u_{1, \xi_{1}}^{*(2 m)}
$$

subject to appropriate boundary conditions

$$
\begin{gathered}
\gamma_{21} u_{1, \zeta}^{*(2 m+2)}+\left(B_{1221}+\bar{p}\right) u_{2, \xi_{1}}^{*(2 m+2)}=0 \quad \text { at } \zeta= \pm 1, \\
\left(B_{2222}+\bar{p}\right) u_{2, \zeta}^{*(2 m+2)}+B_{2233} u_{3, \xi_{3}}^{*(2 m+2)}-\gamma_{23} p_{t}^{*(2 m+2)}=-B_{1122} u_{1, \xi_{1}}^{*(2 m)} \quad \text { at } \zeta= \pm 1, \\
\gamma_{23} u_{3, \zeta}^{*(2 m+2)}=-\left(B_{2332}+\bar{p}\right) u_{2, \xi_{3}}^{*(2 m)} \quad \text { at } \zeta= \pm 1 .
\end{gathered}
$$

The general solution for this system can be written in the form

$$
\begin{aligned}
u_{i}^{*(2 m+2)}= & \sum_{n=0}^{m+1} u_{i}^{*(2 m+2, n)} \zeta^{n} \cos \left(\frac{\pi n}{2}+\frac{\Lambda_{3}^{e} \zeta}{\sqrt{\gamma_{23}}}\right)+\sum_{n=0}^{m+1} U_{i}^{*(2 m+2,2 n)} \zeta^{2 n} \\
& +\sum_{n=0}^{m} v_{i}^{*(2 m+2, n)} \zeta^{n} \cos \left(\frac{\pi n}{2}+\frac{\Lambda_{3}^{e} \zeta}{\sqrt{\gamma_{21}}}\right), \quad i \in\{1,3\}, \\
u_{2}^{*(2 m+2)}= & \sum_{n=0}^{m+1} u_{2}^{*(2 m+2, n)} \zeta^{n} \sin \left(\frac{\pi n}{2}+\frac{\Lambda_{3}^{e} \zeta}{\sqrt{\gamma_{23}}}\right) \\
& +\sum_{n=0}^{m} U_{2}^{*(2 m+2,2 n+1)} \zeta^{2 n+1}+\sum_{n=0}^{m} v_{2}^{*(2 m+2, n)} \zeta^{n} \sin \left(\frac{\pi n}{2}+\frac{\Lambda_{3}^{e} \zeta}{\sqrt{\gamma_{21}}}\right), \\
& +\sum_{n=0}^{m+1} p_{t}^{*(2 m+2, n)} \zeta^{n} \cos \left(\frac{\pi n}{2}+\frac{\Lambda_{3}^{e} \zeta}{\sqrt{\gamma_{23}}}\right) \\
& +\sum_{n=0}^{m+1} P_{t}^{*(2 m+2,2 n)} \zeta^{2 n}+\sum_{n=0}^{m} \tilde{p}_{t}^{*(2 m+2, n)} \zeta^{n} \cos \left(\frac{\pi n}{2}+\frac{\Lambda_{3}^{e} \zeta}{\sqrt{\gamma_{21}}}\right),
\end{aligned}
$$

and corresponding functions $u_{k}^{*(2 m+2, n)}, U_{k}^{*(2 m+2, n)}, v_{k}^{*(2 m+2, n)}, p_{t}^{*(2 m+2, n)}, P_{k}^{*(2 m+2, n)}$ and $\tilde{p}_{k}^{*(2 m+2, n)}$ are to be identified upon insertion into the system (4.82)-(4.88). 


\section{Illustrative numerical results}

Numerical results are now presented in respect of the Varga strain energy function

$$
W\left(\lambda_{1}, \lambda_{2}, \lambda_{3}\right)=\mu\left(\lambda_{1}+\lambda_{2}+\lambda_{3}-3\right)
$$

where $\mu$ is the shear modulus and $\lambda_{m}, m \in\{1,2,3\}$ are the principal stretches of the initial static deformation. For this material, the components of the elasticity tensor can be represented in terms of the principal stretches as follows

$$
B_{i j i j}=\frac{\mu \lambda_{i}^{2}}{\lambda_{i}+\lambda_{j}}, \quad B_{i j j i}=-\frac{\mu \lambda_{i} \lambda_{j}}{\lambda_{i}+\lambda_{j}}, \quad i \neq j, \quad i, j \in\{1,2,3\} .
$$

The material parameters used in figures $1-5$ are summarised in table 1 . Figure 1 shows

(a)

\begin{tabular}{|c|c|c|c|c|c|}
\hline$\mu$ & $\lambda_{1}$ & $\lambda_{2}$ & $\lambda_{3}$ & $\sigma_{2}$ & $\theta$ \\
\hline 2.5 & 0.6 & 1.6 & 1.04 & 1.0 & $15^{\circ}$ \\
\hline
\end{tabular}

(b)

\begin{tabular}{|c|c|c|c|}
\hline$\mu$ & $\lambda_{2}$ & $\sigma_{2}$ & $\theta$ \\
\hline 2.5 & 1.6 & 1.0 & $15^{\circ}$ \\
\hline
\end{tabular}

Table 1: Parameters used in generating graphs. Table (a) contains data for figures 1-3, table (b) specifies parameters used for figures 4-5, unless otherwise stated on graph.

the scaled group velocity $\bar{v}_{g}$ of the first six harmonics (three for each of the two families of cut-off frequencies) for propagation along $\theta=15^{\circ}$ against the scaled wave number $k h, \bar{v}_{g}$ being defined by

$$
\bar{v}_{g}=\frac{\partial \bar{\omega}}{\partial(k h)},
$$

in which $\bar{\omega}$ is the scaled frequency. An interesting feature of this graph is the strongly linear behaviour of the group velocity associated with each harmonics within the low wave number region, especially noticeable for those associated with the second family of cut-off frequencies. To analyse this we consider, as an example, the frequency expansion (3.9), which may be represented as

$$
\bar{\omega}^{2}=\left(\Lambda_{1}^{e}\right)^{2}+\mathcal{E}_{1}^{(2)} \eta^{2}+O\left(\eta^{4}\right), \quad \mathcal{E}_{1}^{(2)}=\mathcal{E}_{1 s}^{(2)} s_{\theta}^{2}+\mathcal{E}_{1 c}^{(2)} c_{\theta}^{2}
$$

Using (5.3) we are able to conclude that

$$
\bar{v}_{g}=\frac{\mathcal{E}_{1}^{(2)}}{\Lambda_{1}^{e}} \eta+O\left(\eta^{3}\right),
$$

indicating low wave number linear variation with an $O\left(\eta^{3}\right)$ correction.

A further interesting feature of figure 1 is the possible existence of negative group velocity at low wave number. For the chosen parameters, this is specifically associated with the first family of cut-off frequencies and is evident in figure 1 for the first three harmonics. Plots depicting the scaled frequencies of these three harmonics, and showing numerical and 


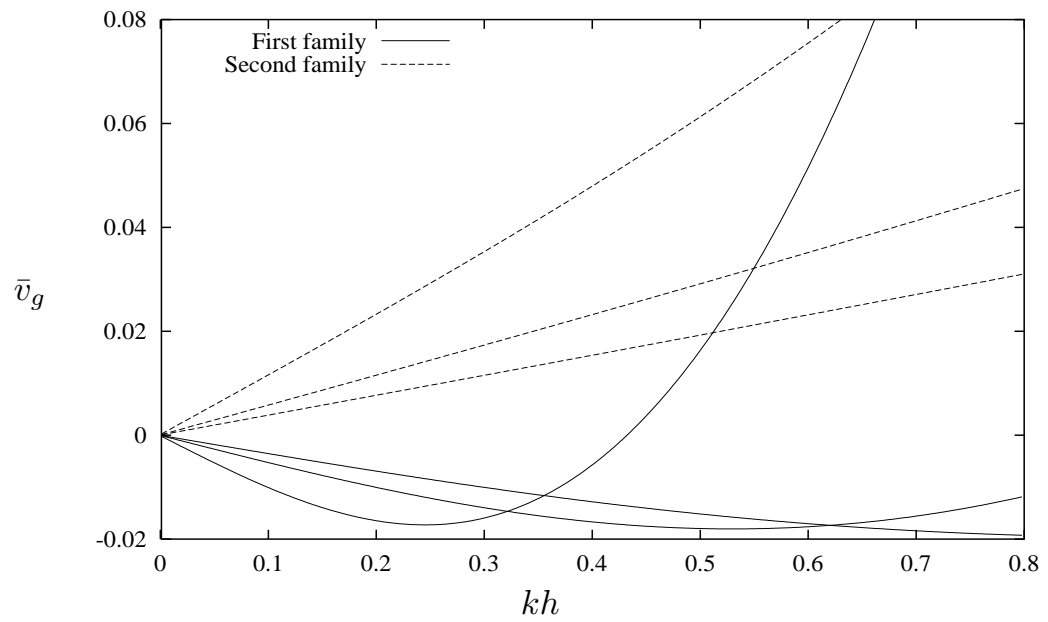

Figure 1: Group velocity for the first six harmonics in low wave number regime, $\theta=15^{\circ}$.
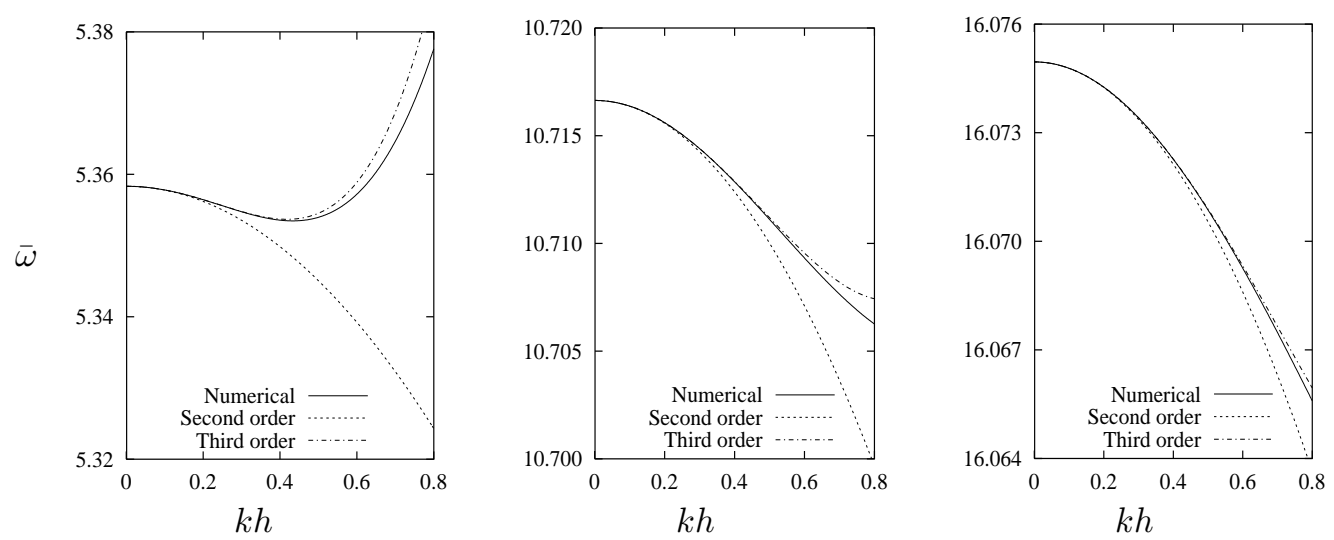

Figure 2: Frequencies of the first three harmonics, associated with the first family of cut-off frequencies.

asymptotic solutions, are presented in figure 2. Negative group velocity corresponds to the decreasing of frequency as the wave number increases and indicates that certain modes may have frequencies below the cut-off. In order for this phenomenon to manifest itself it is necessary that $\mathcal{E}_{1}^{(2)}<0$. When this occurs it is clear that the third order correction term must be used to adequately approximate the numerical solution in all but the very lowest wave number region. We note that in general the asymptotic frequency approximations improve as $n$, the harmonic number, increases, the third order term being $O\left(n^{-2}\right)$. The harmonics associated with the second family of cut-off frequencies have no negative group velocity. For these the second order approximation is almost indistinguishable from the third order, as illustrated in figure 3.

As previously mentioned, the existence of negative group velocity is connected with the change of sign of $\mathcal{E}_{1 c}^{(2)}$ or $\mathcal{E}_{2 s}^{(2)}$ in the governing equation (4.33) or (4.76), respectively. 

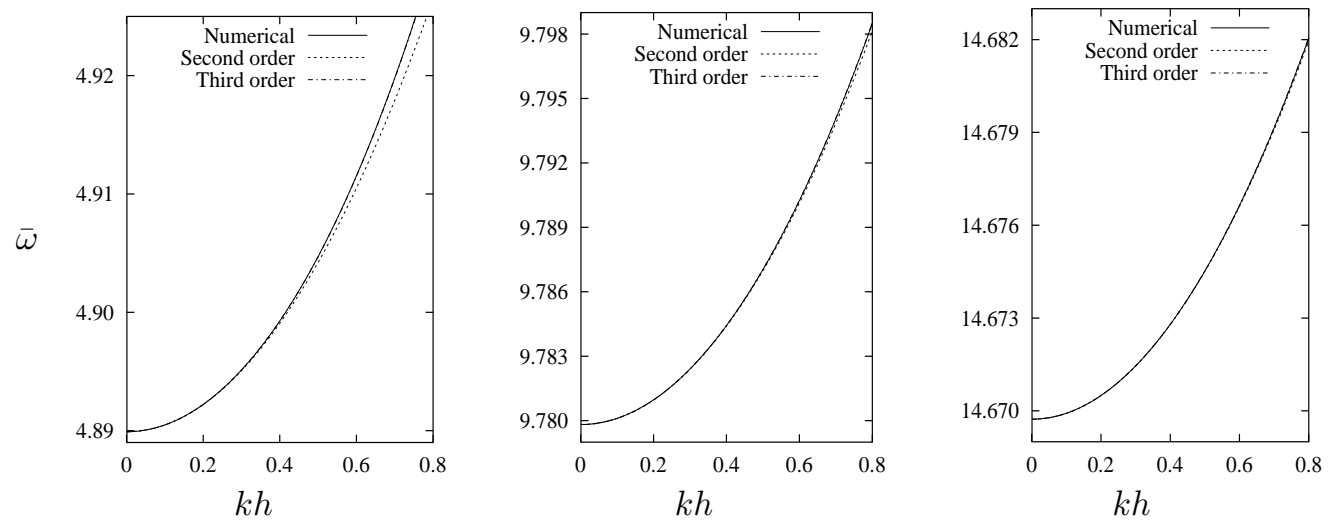

Figure 3: Frequencies of the first three harmonics, associated with the second family of cut-off frequencies.
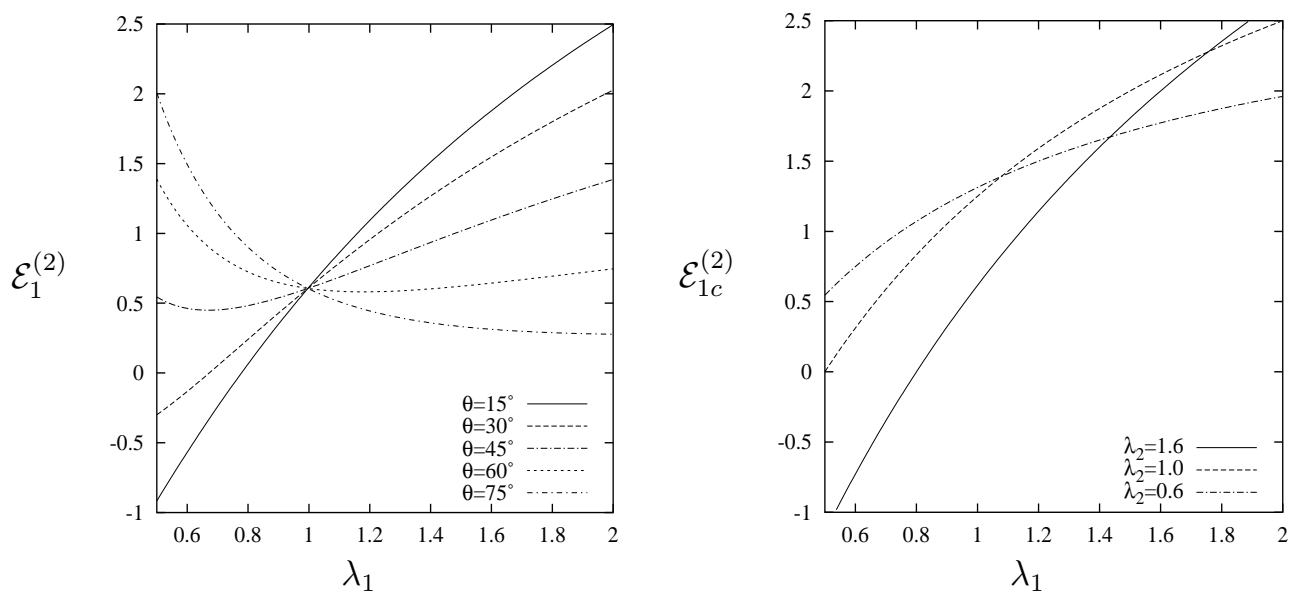

Figure 4: Dependence of the second order frequency expansion term $\mathcal{E}_{1}^{(2)}$ and governing equation coefficient $\mathcal{E}_{1 c}^{(2)}$ on the change of in-plane deformation, shown for the motions associated with the first family of cut-off frequencies.

When this occurs there is a significant change in the character of the appropriate governing equation. In particular, time and one of the in-plane spatial variables swap roles. In fact, it was shown by Kaplunov et al. (2000b) that in the plain strain case a similar change of the coefficient sign changes the analogous equation from hyperbolic to elliptic. In the plain strain case this change of sign is always synonymous with the existence of negative group velocity. However, in the present case it is quite possible for time and one of the in-plane spatial variables to swap roles and the group velocity remain positive, see figure 4 . In this figure the left hand graph shows the dependence of the second order term of the frequency expansion on in-plane deformation, with fixed normal deformation. The sign of this term indicates the sign of the group velocity, see equation (5.5). We note that this phenomenon 

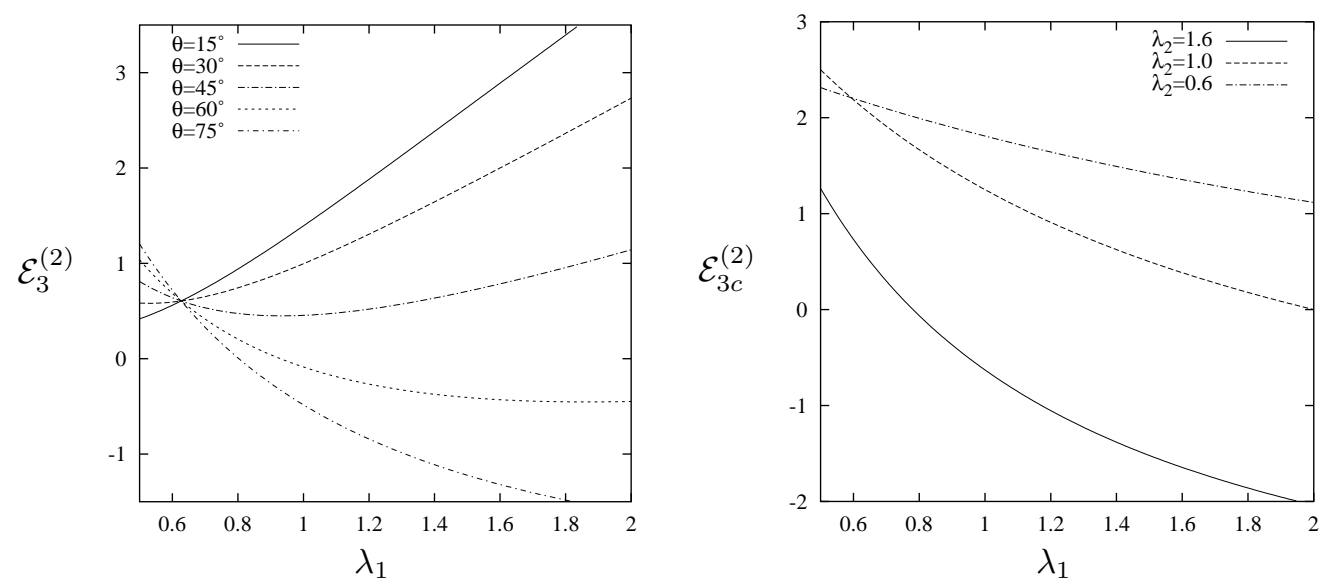

Figure 5: Dependence of the second order frequency expansion term $\mathcal{E}_{3}^{(2)}$ and governing equation coefficient $\mathcal{E}_{3 c}^{(2)}$ on the change of in-plane deformation, shown for the motions associated with the second family of cut-off frequencies.

is most likely to occur for propagation along a principal direction. The right hand graph shows the behaviour of $\mathcal{E}_{1 c}^{(2)}$, the sign of which triggers the role change of the spatial variable and time previously mentioned. Since $\mathcal{E}_{1 c}^{(2)}$ is independent of $\theta$, and $\mathcal{E}_{1 s}^{(2)}$ always positive, a value of $\theta$ may always be chosen such that $v_{g}>0$.

Similar comments may also be made for the coefficients associated with the second family of cut-off frequencies, corresponding numerical illustrations being shown in figure 5 . For example, an initial pre-stress arising from the principal stretches $\lambda_{1}=1, \lambda_{2}=1.6$ renders the coefficient $\mathcal{E}_{3 c}^{(2)}$ negative, see the right hand graph, the governing equation not being a typical hyperbolic equation. However, the left hand graph shows that for the angles of propagation $\theta \geqslant 45^{\circ}$ the group velocity of the harmonics still remain positive.

\section{Acknowledgement}

The work of A. V. Pichugin is supported by the U.K. Overseas Research Student Award and by the University of Salford. These awards are very gratefully Acknowledged.

\section{References}

Achenbach, J. D., 1969, An asymptotic method to analyse the vibrations of an elastic layer. Trans. ASME Jl. Appl. Mech., 36, 65-72.

Dowaikh, M. A. \& Ogden, R. W., 1990, On surface waves and deformations in a prestressed incompressible elastic solid. IMA Jl. Appl. Math., 44, 261-284. 
Dowaikh, M. A. \& Ogden, R. W., 1991, Interfacial waves and deformations in prestressed elastic media. Proc. R. Soc. Lond. A., 433, 313-328.

Goldenveiser, A. L., 1962, An application of asymptotic intergartion of the equations of elasticity to derive an approximate theory for plate bending. PMM (Appl. Math. Mech.), 26, 668-686.

Goldenveiser, A. L., 1966, The principles of reducing three-dimensional problems of elasticity to two-dimensional problems of the theory of plates and shells. In Applied Mechanics, Proc. 11th Int. Congr. Appl. Mech. (H. Gortler, ed.), Springer: Berlin, pp. $306-311$.

Kaplunov, J. D., 1989, Equations for high-frequency long-wave vibrations of an elastic layer lying on an acoustic half-space. Doklady Akad. Nauk SSSR (Soviet Phys. Dokl.), 309, 1077-1081.

KAPlunOV, J. D., 1990, High-frequency stress-strain states of small variability in elastic shells. Izv. Akad. Nauk SSSR, Ser. Mekhanika Tverdogo Tela (Mechanics of Solids), 25(5), 147-157.

Kaplunov, J. D., 1995, Long wave vibrations of a thin body with fixed faces. Quart. Jl. Mech. Appl. Math., 48(3), 311-327.

Kaplunov, J. D., Kossovich, L. Y., \& Nolde, E. V., 1998, Dynamics of thin walled elastic bodies. New York: Academic Press.

Kaplunov, J. D., Kossovich, L. Y., \& Rogerson, G. A., 2000a, Direct asymptotic integration of the equations of transversely isotropic elasticity for a plate near cut-off frequencies. Quart. J. Mech. Appl. Math., 53, 323-341.

Kaplunov, J. D. \& Markushevich, D. G., 1993, Plane vibrations and radiation of an elastic layer lying on a liquid half-space. Wave Motion, 17, 199-211.

Kaplunov, J. D., Nolde, E. V., \& Rogerson, G. A., 2000b, An asymptotically consistent model for long wave high frequency motion in a pre-stressed elastic plate. Mech. Math. Solids, to appear.

Kaplunov, J. D., Nolde, E. V., \& Rogerson, G. A., 2000c, A low frequency model for dynamic motion in pre-stressed incompressible elastic structures. Proc. R. Soc. Lond. A., 456, 2589-2610.

Ogden, R. W., 1984, Non-linear Elastic Deformations. New York: Ellis Horwood. 
Ogden, R. W. \& Roxburgh, D. G., 1993, The effect of pre-stress on the vibration and stability of elastic plates. Int. Jl. Eng. Sci., 30, 1611-1639.

Ogden, R. W. \& Sotiropoulos, D. A., 1995, On interfacial waves in pre-stressed layered incompressible solids. Proc. R. Soc. Lond. A., 450, 319-341.

Rogerson, G. A., 1997, Some asymptotic expansions of the dispersion relation for an incompressible elastic plate. Int. Jl. Solid. Struct., 34(22), 2785-2802.

Rogerson, G. A. \& Fu, Y. B., 1995, An asymptotic analysis of the dispersion relation of a pre-stressed incompressible elastic plate. Acta Mechanica, 111, 59-77.

Rogerson, G. A. \& SAndiford, K. J., 1997, Flexural waves in pre-stressed incompressible elastic composites. Quart. Jl. Mech. Appl. Math., 50(4), 597-624.

Rogerson, G. A. \& SAndiford, K. J., 1999, Harmonic wave propagation along a nonprincipal direction in a pre-stressed elastic plate. Int. Jl. Eng. Sci., 37, 1663-1691.

Sheridan, P. M., James, F. O., \& Miller, T. S., 1992, Design of components. In Engineering with rubber (A. N. Gent, ed.), Munich: Hanser, pp. 209-235. 